\title{
Bayesian Algorithmic Mechanism Design
}

\author{
Jason D. Hartline* $\quad$ Brendan Lucier ${ }^{\ddagger}$
}

September 3, 2018

\begin{abstract}
The principal problem in algorithmic mechanism design is in merging the incentive constraints imposed by selfish behavior with the algorithmic constraints imposed by computational intractability. This field is motivated by the observation that the preeminent approach for designing incentive compatible mechanisms, namely that of Vickrey, Clarke, and Groves; and the central approach for circumventing computational obstacles, that of approximation algorithms, are fundamentally incompatible: natural applications of the VCG approach to an approximation algorithm fails to yield an incentive compatible mechanism. We consider relaxing the desideratum of (ex post) incentive compatibility (IC) to Bayesian incentive compatibility (BIC), where truthtelling is a Bayes-Nash equilibrium (the standard notion of incentive compatibility in economics). For welfare maximization in single-parameter agent settings, we give a general black-box reduction that turns any approximation algorithm into a Bayesian incentive compatible mechanism with essentially the same ${ }^{1}$ approximation factor.
\end{abstract}

\section{Introduction}

Can any approximation algorithm be converted into an approximation mechanism for selfish agents? This question is framed by a fundamental incompatibility between the standard economic approach for the design of mechanisms for selfish agents (the Vickrey-Clarke-Groves (VCG) mechanism) and the standard algorithmic approach for circumventing computational intractability (approximation algorithms). The conclusion from this incompatibility, driving much of the field of algorithmic mechanism design, is that incentive and algorithmic constraints must be dealt with simultaneously (See e.g., [17]). For a large, important class of problems, we arrive at the opposite conclusion: there is a general approximation-preserving reduction from mechanism design to algorithm design!

The goal of mechanism design is to construct the rules for a system of agents so that in the equilibrium of selfish agent behavior a desired objective is obtained. For settings of incomplete information the standard game theoretic equilibrium concept is Bayes-Nash equilibrium (BNE), which is defined by mutual best response when the prior distribution of agent payoffs is common knowledge. The revelation principle [20] suggests that when looking for mechanisms with desirable Bayes-Nash equilibria, one must look no further than those with truthtelling as a Bayes-Nash

\footnotetext{
* Supported in part by NSF Grant CCF-0830773 and NSF Career Award CCF-0846113.

${ }^{\dagger}$ Northwestern University, Evanston, IL, USA. email: hartline@eecs.northwestern.edu

${ }^{\ddagger}$ University of Toronto, Toronto, ON, Canada. email: blucier@cs.toronto.edu

${ }^{1}$ More specifically, we obtain a polynomial time approximation scheme with an $\epsilon$ loss that is either additive or multiplicative, depending on the problem setting. This error term arises from statistical methods that seem necessary for a black-box reduction.
} 
equilibrium, also known as Bayesian incentive compatible (BIC) mechanisms. Almost all of the computer science literature has focused on the BIC subclass of ex post incentive compatible (IC) mechanisms where truthtelling is a dominant strategy. While IC is aesthetically appealing because it is congruous with worst-case-style results, it is not generally without loss!

This loss is evident in the computer science theory of IC mechanism design, which is described most characteristically by impossibility. For instance, for single-minded combinatorial auctions of $m$ items, the optimal worst-case approximation factor (under standard complexity theoretic assumptions) is $\sqrt{m}$ [19]. With such strong lower bounds, a relevant theory must make relaxations. For many problems within the realm of computer systems; e.g., online auctions (eBay), advertising auctions (Google, Yahoo!, MSN), file sharing (BitTorrent), routing (TCP/IP), scheduling (SETI@home), and video streaming (YouTube); high volume should enable demand distributions to be estimated. With demand distributions, the natural algorithmic and mechanism design problems are Bayesian.

Agent incentives in Bayesian mechanism design are very well understood in single-parameter settings, where each agent has a single independent private value for receiving a service (see, e.g, [20]). For the single parameter setting it is known that a mechanism is BIC if and only if (a) the probability an agent is served (a.k.a. the allocation rule) is monotone non-decreasing in the agent's value for service, and (b) the agent's expected payment (a.k.a. the payment rule) is of a particular form identified precisely from the allocation rule. ${ }^{2}$

The main challenge in reducing BIC (or IC) mechanism design to algorithm design is that approximation algorithms do not generally have monotone allocation rules. Our reduction shows that in a Bayesian setting we can convert any non-monotone allocation rule into a monotone one without compromising its social welfare. The main technical observation that enables this reduction is that, in a Bayesian setting, we can focus on a single agent for whom the allocation rule is not monotone, apply a transformation that fixes this non-monotonicity (and weakly improves our objective), and no other agents are affected (in a Bayesian sense). Therefore, we can apply the transformation independently to each agent. Our reduction is as follows:

1. For each agent, identify intervals in which the agent's allocation rule is non-monotone. (This is a property of the distribution and algorithm and can be done prior to considering any agent bids.)

2. For each agent, if their bid falls in an (above identified) interval, redraw the agent's bid from the prior distribution conditioned on being within the interval.

3. Run the approximation algorithm on the resulting bids and output its solution.

Notice that under the assumption that the original values are drawn according to the common prior, the redrawing of values does not alter this prior.

Three items must be clarified. First, there are many ways one might try to choose intervals in Step 1 of the reduction and most of them are incompatible with mechanism design. To address this issue, we develop a monotonizing technique for allocation rules (adapted from the standard ironing procedure from the field of Bayesian optimal mechanism design [20]). Second, we are unlikely to have access the functional form of the allocation rule. To address this issue, we estimate the allocation rule by sampling the distribution and making black-box calls to the algorithm. These estimates

\footnotetext{
${ }^{2}$ Probabilities and expectations above are taken with respect to both the distribution of agent values and possible randomization in the mechanism.
} 
can be made precise enough to enable arbitrary small loss in welfare (i.e., a fully polynomial time approximation scheme). Finally, we must also determine payments for our monotonized allocation rule. For this, a general approach of Archer et al. [3] suffices.

Our results apply generally to single-parameter agent settings where the designer's objective is to maximize the social welfare (e.g., single-minded combinatorial auctions). In the most general form, such an algorithmic problem can be written as finding an allocation $\mathbf{x}=\left(x_{1}, \ldots, x_{n}\right)$ to maximize $\sum_{i} v_{i} x_{i}-c(\mathbf{x})$ for agent valuations $\mathbf{v}=\left(v_{1}, \ldots, v_{n}\right)$ and cost function $c(\cdot)$. For instance, the multicast auction problem of Feigenbaum et al. [14] is the special case where the $c(\mathbf{x})$ is the sum of the costs of tree edges necessary to connect all agents served by $\mathbf{x}$ to the root (generally, the Steiner tree problem). A special and relevant case occurs when costs are zero for $\mathbf{x}$ in some feasible set system $\mathcal{X}$ and all other allocations are infeasible (i.e, $c(\mathbf{x})=0$ if $\mathbf{x} \in \mathcal{X}$ and $\infty$ otherwise). For the single-item auction, $\mathcal{X}$ is the collection of all sets of cardinality at most one; and for singleminded combinatorial auctions, $\mathcal{X}$ contains all sets of agents with non-intersecting desired bundles. Our most general result does not need any restrictions on the cost function or the set system. In particular, costs can be arbitrarily non-monotone or the set system non-downward-closed (e.g. public good or scheduling problems).

For any $\epsilon$, we give a black box reduction that, in polynomial time in the number of agents and $1 / \epsilon$, converts any approximation algorithm into a BIC mechanism with an additive loss of $\epsilon$ to the social welfare. We also give a pseudo-polynomial time reduction to a BIC mechanism with a multiplicative loss of $\epsilon$, and a fully polynomial time approximation scheme for the special case of downward-closed feasibility problems. Thus, the approximation complexity of social welfare in single-parameter settings is the same for algorithms and BIC mechanisms.

For the most studied single-parameter mechanism design problems, the performance of the best ex post IC approximation mechanism matches the best approximation algorithm (e.g., singleminded combinatorial auctions [19] and related machine scheduling [13]). None-the-less, our approach gives the best known BIC approximation mechanism for many problems, such as auctions under various graph constraints [2] and auctions of convex bundles [5].

Our result demonstrates that there is no gap between algorithmic approximation and approximation by BIC mechanisms. The remaining (theoretical) question of gaps in approximation factors imposed by incentive constraints is thus focused on whether BIC is more powerful than IC for social welfare maximization. For other non-welfare-maximization objectives (e.g., makespan) the question of a general reduction remains open.

Related Work The design of ex post IC mechanisms for social welfare problems is well studied, notably for the specific settings of combinatorial auctions [3, 5, 18, 19]. Lehmann et al. [19] introduced the problem of polynomial time approximation of social welfare for single-minded combinatorial auctions and give a mechanism that matches the best algorithmic approximation factor. Archer et al. [3] considered the setting where there are many (at least logarithmic) copies of each item and gave a $(1+\epsilon)$-approximation mechanism. Archer and Tardos [4] gave a (single-parameter) related machine scheduling mechanism that approximates the makespan. Dhangwatnotai et al. [13] gave a mechanism for related machine scheduling that approximates makespan and matches the algorithmic lower bound. All of the above results are for ex post incentive compatible mechanisms.

There has been a large literature on multi-parameter combinatorial auctions and approximation, but this is only tangentially related to our work so we do not cite it exhaustively.

The literature contains a few reductions from mechanism design to algorithm design of varying 
degrees of generality. Lavi and Swamy [18] consider IC mechanisms for multi-parameter packing problems and give a technique for constructing a (randomized) $\beta$-approximation mechanism from any $\beta$-approximation algorithm that verifies an integrality gap. Babaioff et al. [6] look at the equilibrium notion of algorithmic implementation in undominated strategies and gives a technique for turning a $\beta$-algorithm into a $\beta\left(\log v_{\max }\right)$-approximation mechanism. This solution concept requires that no agent plays a strategy that is dominated by an easy to find strategy. Their approach applies to single-valued combinatorial auctions and does not require the mechanism to know which bundles each agent desires.

There have been a few related studies of Bayes-Nash equilibrium. Christodoulou et al. [12] consider Bayes-Nash equilibria of simultaneous Vickrey auctions in a combinatorial setting and show that these give a 2-approximation when agents' valuations are submodular. Gairing et al. [15] consider Bayes-Nash equilibria of a routing game and study worst-case performance. Borodin and Lucier [9] study worst-case performance of Bayes-Nash equilibria in combinatorial mechanisms based on greedy algorithms.

There are many papers on profit maximization that consider Bayesian design settings. These papers do not tend to consider computational constraints and for many of these (non-computational) settings the restriction to ex post incentive compatibility is without loss. ${ }^{3}$ One notable exception is Bhattacharya et al. [8] which focuses on the problem of selling heterogeneous goods to agents with linear valuations. They construct a polynomial time 4-approximation mechanism. Their approximation result requires that the type distributions satisfy the monotone hazard rate assumption. In a spirit similar to this paper, they make heavy use of the Bayesian setting to obtain a polynomial runtime.

Organization We describe in detail the model for single-parameter agents, Bayesian approximation, Bayesian incentive compatibility, and foundational economic theory in Section 2. In Section 3 we give the reduction in an ideal setting where the allocation rule of the algorithm for the given distribution on agent values is precisely known. This reduction is lossless. In Section 4 we develop the reduction in the black-box model where we must sample the distribution and run the algorithm to determine its allocation. Conclusions and open problems are discussed in Section 5.

\section{Model and Definitions}

Algorithms We consider algorithms for binary single-parameter agent settings. An algorithm in such a setting must select a set of agents to serve. This allocation is denoted by $\mathbf{x}=\left(x_{1}, \ldots, x_{n}\right)$ where $x_{i}$ is an indicator for whether or not agent $i$ is served. Agent $i$ has valuation $v_{i}$ for being served. Without loss for non-negative bounded-support distributions, we will assume $v_{i} \in[0,1] .{ }^{4}$ The vector $\mathbf{v}=\left(v_{1}, \ldots, v_{n}\right)$ of valuations is the valuation profile.

In the general costs setting, the seller may have some cost function $c(\cdot)$ over allocations representing the cost of serving the allocated set (e.g., Steiner tree problems [14]). The general feasibility setting is the special case where costs are zero (feasible) or infinity (infeasible). These include scheduling and public good problems. An important subclass are downward-closed settings where

\footnotetext{
${ }^{3}$ One non-computational setting where ex post incentive compatibility is with loss is when the profit maximizing seller has a strict no-deficit constraint [11].

${ }^{4}$ The bounded support assumption is unnecessary except for our results using sampling, where we believe it is realistic.
} 
any subset of a feasible set is feasible. Downward closed settings include single-minded combinatorial auctions [19] and knapsack auctions [1].

An algorithm $\mathcal{A}$ is simply an allocation rule that maps valuation profiles to allocations. The allocation rule for $\mathcal{A}$ we will denote by $\mathbf{x}(\mathbf{v})$. Our objective is the social welfare which is $\mathcal{A}(\mathbf{v})=$ $\sum_{i} v_{i} x_{i}(\mathbf{v})-c(\mathbf{x}(\mathbf{v}))$. We allow $\mathcal{A}$ to be randomized in which case $x_{i}(\mathbf{v})$ is a random variable; $\mathcal{A}(\mathbf{v})$ denotes the expected welfare of the algorithm for valuation profile $\mathbf{v}$. OPT(v) will denote the maximum social welfare.

We will consider these algorithmic problems in a Bayesian (a.k.a. stochastic) setting where the valuations of the agents are drawn from a product distribution $\mathbf{F}=F_{1} \times \cdots \times F_{n}$. Agent $i$ 's cumulative distribution and density functions are denoted $F_{i}$ and $f_{i}$, respectively. The distribution is assumed to be common knowledge to the agents and designer.

The pair $(c(\cdot), \mathbf{F})$ defines a setting for single-parameter algorithm design which we will take as implicit. For this setting, the optimal expected welfare is OPT $=\mathbf{E}_{\mathbf{v} \sim \mathbf{F}}[\mathrm{OPT}(\mathbf{v})]$ and the algorithm's expected welfare is $\mathcal{A}=\mathbf{E}_{\mathbf{v} \sim \mathbf{F}}[\mathcal{A}(\mathbf{v})]$. An algorithm is a worst-case $\beta$-approximation if for all $\mathbf{v}, \mathcal{A}(\mathbf{v}) \geq \operatorname{OPT}(\mathbf{v}) / \beta$. An algorithm is a Bayesian $\beta$-approximation if $\mathcal{A} \geq \mathrm{OPT} / \beta$.

Mechanisms A mechanism $\mathcal{M}$ consists of an allocation rule and a payment rule. We denote by $\mathbf{x}(\mathbf{v})$ and $\mathbf{p}(\mathbf{v})$ the allocation and payment rule of an implicit mechanism $\mathcal{M}$. We assume agents are risk neutral and individually desire to maximize their expected utilities. Agent $i$ 's utility for allocation $\mathbf{x}$ and payments $\mathbf{p}$ is $v_{i} x_{i}-p_{i}$. We consider single-round, sealed-bid mechanisms where agents simultaneously bid and the mechanism then computes the allocation and payments.

Our goal is a mechanism that has good social welfare in equilibrium. The standard economic notion of equilibrium for games of incomplete information is Bayes-Nash equilibrium (BNE). The revelation principle says that any equilibrium that is implementable in BNE is implementable with truthtelling as the BNE strategies of the agents. ${ }^{5}$ Meaning: an agent that believes the other agents are reporting their values truthfully as given by the distribution has a best response of also reporting truthfully. A mechanism with truthtelling as a BNE is Bayesian incentive compatible (BIC). ${ }^{6}$

It will be useful to consider agent $i$ 's expected payment and probability of allocation conditioned on their value. To this end, denote $p_{i}\left(v_{i}\right)=\mathbf{E}_{\mathbf{v}, \mathcal{A}}\left[p_{i}(\mathbf{v}) \mid v_{i}\right]$ and $x_{i}\left(v_{i}\right)=\mathbf{E}_{\mathbf{v}, \mathcal{A}}\left[x_{i}(\mathbf{v}) \mid v_{i}\right]$. The following theorem characterizes BIC mechanisms.

Theorem 2.1 [20] A mechanism is BIC if and only if for all agents $i$ :

- $x_{i}\left(v_{i}\right)$ is monotone non-decreasing, and

- $p_{i}\left(v_{i}\right)=v_{i} x_{i}\left(v_{i}\right)-\int_{0}^{v_{i}} x_{i}(z) d z+p_{i}(0)$.

Usually, $p_{i}(0)$ is assumed to be zero.

This motivates the following definition:

Definition 2.1 An allocation rule $\mathbf{x}(\cdot)$ is monotone for distribution $\mathbf{F}$ if $x_{i}\left(v_{i}\right)$ is monotone nondecreasing for all $i$. An algorithm is monotone if its allocation rule is monotone.

\footnotetext{
${ }^{5}$ The revelation principle holds even in computational settings; any BNE for which the agent strategies and the mechanism can be computed in polynomial time can be converted into a polynomial time BIC mechanism.

${ }^{6} \mathrm{Much}$ of the computer science literature on mechanism design focuses on dominant strategy equilibrium (DSE) and ex post incentive compatibility (IC). This is not without loss in many settings and therefore should be considered with care when addressing computational questions in mechanism design.
} 
From Theorem 2.1, BIC and monotone are equivalent and we will use them interchangeably for both algorithms and mechanisms, though we will prefer "BIC" when the focus is incentive properties and "monotone" when the focus is algorithmic properties.

Computation Our main task in demonstrating that the approximation complexity of algorithms and BIC mechanisms is the same by giving an approximation-preserving reduction from the BIC mechanism design problem to the algorithm design problem. In other words, we use the algorithm's allocation rule to compute the mechanism's allocation and payment rules. As we are in a Bayesian setting this computation will also need access to the distribution.

We consider two models of computation: an ideal model and a black-box model. In the ideal model, we will assume we have explicit access to the functional form of the distribution and allocation rule and we will assume we can perform calculus on these functions. While this model is not realistic, we present it for the sake of clarity in explaining the economic theory that drives our results. In the black-box model we will assume we can query the algorithm on any input and that we can sample from the distribution on any subinterval of its support. Our philosophy is that the ideal model is predictive of what is implementable in polynomial time and we verify this philosophy by instantiating approximately the same reduction under the black-box model.

\section{Reduction: Ideal Model}

In this section we prove that, in the ideal model, any Bayesian algorithm can be made BIC without loss of performance.

Theorem 3.1 In the ideal model and general cost settings, a BIC algorithm $\overline{\mathcal{A}}$ can be computed from any algorithm $\mathcal{A}$. Its expected social welfare satisfies $\overline{\mathcal{A}} \geq \mathcal{A}$.

Theorem 3.1 implies an immediate corollary for Bayesian approximation.

Corollary 3.2 In the ideal model and general cost settings, a BIC Bayesian $\beta$-approximation $\overline{\mathcal{A}}$ can be computed from any Bayesian $\beta$-approximation algorithm, $\mathcal{A}$.

Corollary 3.2 applies in the special case that $\mathcal{A}$ is a worst-case $\beta$-approximation, but the resulting BIC algorithm $\overline{\mathcal{A}}$ will not necessarily be a worst-case $\beta$-approximation. See Appendix $\mathrm{C}$ for a concrete example.

Let us build some intuition for the requirements of Theorem 3.1. Suppose that we are given an algorithm $\mathcal{A}$ that is monotone for the distribution $\mathbf{F}$. Then $\mathcal{A}$ is already BIC and specifies the allocation rule $\mathbf{x}(\cdot)$, so we must only compute the payment rule. In our ideal model this is trivial given the formula from Theorem 2.1.

Now suppose we have a non-monotone Bayesian $\beta$-approximation algorithm $\mathcal{A}$ with allocation rule $\mathbf{x}(\cdot)$. We would like to use $\mathcal{A}$ to construct a monotone algorithm $\overline{\mathcal{A}}$ from which we can obtain a BIC mechanism by simply computing the payment rule as above. We must make sure that in doing so we do not reduce the algorithm's expected welfare. The key property of our approach which makes it tractable is that we monotonize each agent's allocation rule independently without changing (in a Bayesian sense) the allocation rule any other agent faces. This property is also important for the approximation factor as $\mathcal{A}$ is guaranteed to be a Bayesian $\beta$-approximation only for the given distribution $\mathbf{F}$, and may not be a good approximation for some other distribution. 


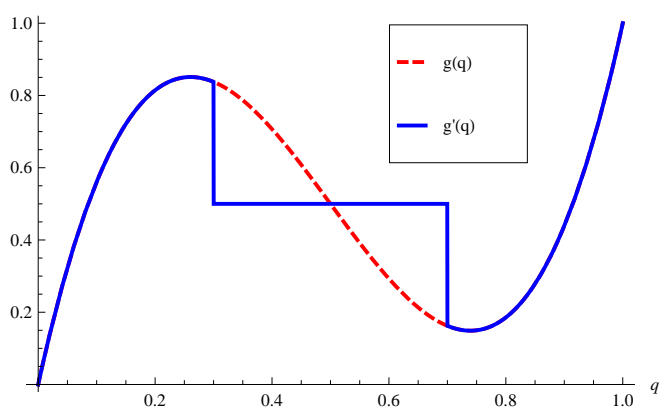

(a)

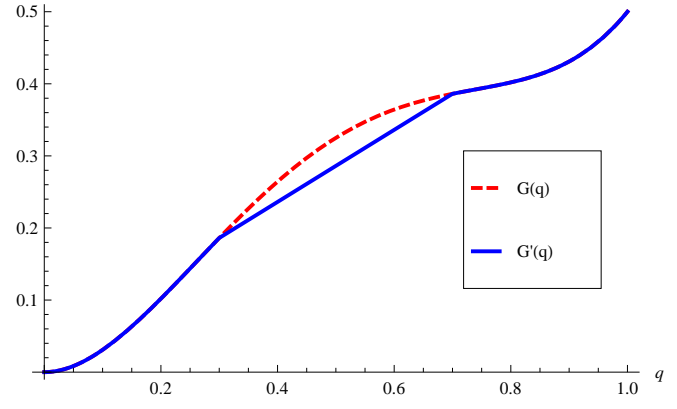

(b)

Figure 1: (a) A non-monotone ironing $g^{\prime}$ (solid) of curve $g$ (dashed). (b) The corresponding integral curves $G^{\prime}$ (solid) and $G$ (dashed) in probability space.

In summary, the desiderata for monotonizing agent $i$ are:

D1. monotone $\bar{x}_{i}\left(v_{i}\right)$,

D2. (weakly) improved social welfare $\mathbf{E}_{v_{i}}\left[v_{i} \bar{x}_{i}\left(v_{i}\right)\right] \geq \mathbf{E}_{v_{i}}\left[v_{i} x_{i}\left(v_{i}\right)\right]$, and

D3. other agents unaffected.

Notice that if we satisfy the last condition we can apply the process simultaneously to all agents.

\subsection{Ironing via Resampling}

There is a history of fixing non-monotonicities in Bayesian mechanism design. Myerson invented the technique of ironing which relies on the fact that if an allocation rule is constant over some interval then any agent within that interval is effectively equivalent to a canonical "average" agent from that interval. Myerson applied this theory to iron virtual valuation functions which are used in Bayesian profit maximization [20]. We will apply this theory directly to allocation rules.

Before we describe our ironing procedure in full, let us develop some more intuition. Suppose allocation rule $x_{i}(\cdot)$ of $\mathcal{A}$ is non-monotone for agent $i$. A simple approach to flattening nonmonotonicities is to choose some interval $[a, b]$ on which $x(\cdot)$ is non-monotone, and to treat the agent identically whenever on this interval. For example, whenever $v_{i} \in[a, b]$ we could choose to pretend that $v_{i}$ is actually some other fixed value $v^{\prime}\left(\right.$ e.g. $\left.v^{\prime}=a\right)$ and pass this "pretend" value $v^{\prime}$ to the algorithm. Unfortunately, if we take this naïve approach, we would have changed the distribution of agent $i$ 's input to the algorithm (in particular, the probability of value $v^{\prime}$ would be increased) and violated D3. In order to maintain D3 we make a minor modification: instead of picking a fixed $v^{\prime}$, we will draw $v^{\prime}$ from $F_{i}$ restricted to the interval $[a, b]$. Thus, we are replacing $v_{i} \in[a, b]$ with $v^{\prime}$ drawn from the same distribution. Other agents cannot tell the difference this operation does not change the distribution of agent $i$ 's input! Moreover, agent $i$ will indeed be treated identically whenever $v_{i} \in[a, b]$ : the new probability of allocation will be precisely the distribution weighted average of $x_{i}(\cdot)$ over the interval $[a, b]$.

Let $x_{i}{ }^{\prime}(\cdot)$ represent the allocation rule obtained from the following procedure (where $\mathbf{v}_{-i} \sim \mathbf{F}_{-i}$ ):

- if $v_{i} \in[a, b]$, redraw $v^{\prime} \sim F_{i}$ restricted to $[a, b]$; else, set $v^{\prime}=v_{i}$. 


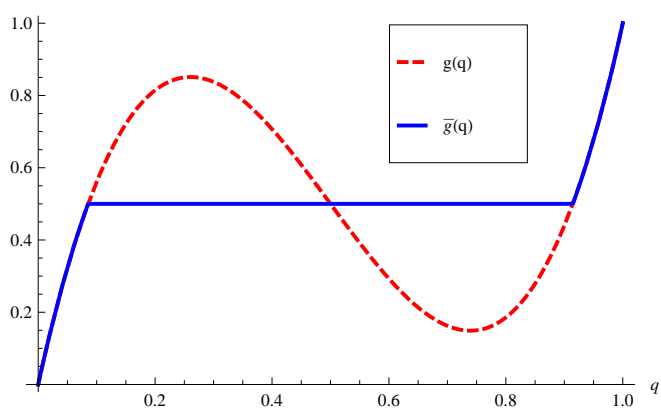

(a)

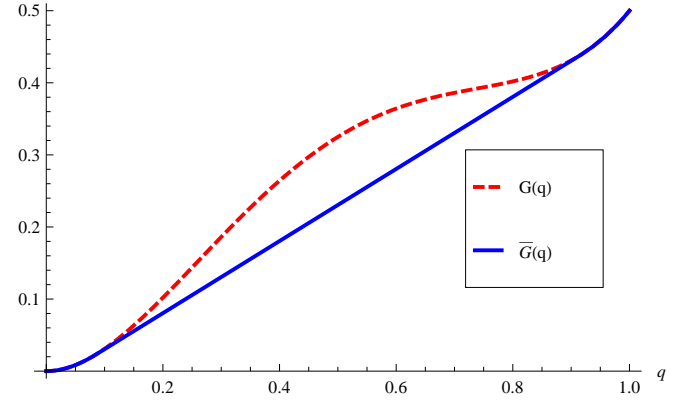

(b)

Figure 2: (a) A monotone ironing $\bar{g}$ (solid) of curve $g$ (dashed). (b) The corresponding integral curves $\bar{G}$ (solid) and $G$ (dashed) in probability space. Note $\bar{G}$ is the convex hull of $G$.

- $\operatorname{run} \mathcal{A}\left(v^{\prime}, \mathbf{v}_{-i}\right)$.

We say that $x_{i}{ }^{\prime}(\cdot)$ is the curve $x_{i}(\cdot)$ ironed on interval $[a, b]$. We note that $x_{i}{ }^{\prime}\left(v_{i}\right)=x_{i}\left(v_{i}\right)$ for $v_{i} \notin[a, b]$ and $x_{i}{ }^{\prime}\left(v_{i}\right)=\mathbf{E}_{v^{\prime} \sim F_{i}}\left[x_{i}\left(v^{\prime}\right) \mid v^{\prime} \in[a, b]\right]$ otherwise. Note that we can easily iron along multiple disjoint intervals, redrawing $v^{\prime}$ from whichever interval contains $v_{i}^{\prime}$ (if any).

We now explore a method for choosing intervals on which to iron in order to obtain monotonicity. It will be instructive to consider the allocation rule in probability space instead of valuation space, and the cumulative allocation rule (also in probability space).

- Let $g(q)=x_{i}\left(F_{i}^{-1}(q)\right)$ be the allocation rule in probability space.

- Let $G(q)=\int_{0}^{q} g(z) d z$ be the cumulative allocation rule.

Notice that monotonicity of $x_{i}(\cdot)$ is equivalent to monotonicity of $g(\cdot)$ which is equivalent to convexity of $G(\cdot)$.

Let $x_{i}{ }^{\prime}(\cdot)$ be $x_{i}(\cdot)$ ironed along some interval $[a, b]$, and consider the corresponding curves $g^{\prime}(\cdot)$ and $G^{\prime}(\cdot)$. This ironing procedure corresponds to replacing $g(\cdot)$ with its average on $[a, b]$, or equivalently $G(\cdot)$ with the line segment connecting $G(F(a))$ to $G(F(b))$ (See Figure 1). ${ }^{7}$ This latter line segment interpretation suggests that we can view our interval selection problem as the problem of replacing portions of curve $G$ with straight line segments so that the resulting curve $\bar{G}$ will be convex. This is precisely the problem of finding the convex hull of $G$ ! Thus the choice of intervals that monotonizes $x_{i}(\cdot)$ (satisfying D1) is precisely the set of intervals defined by the convex hull of $G(\cdot)$. See Figure 2 .

Finally, since the convex hull of $G(\cdot)$ lies below $G(\cdot)$, this transformation weakly improves welfare (satisfying D2). Informally speaking, in moving from cumulative allocation rule $G(\cdot)$ to $\bar{G}(\cdot)$, we lower the probability of low-value allocations in exchange for a corresponding increase in the probability that higher-valued allocations occur. This intuition is made more precise in Lemma 3.4, below.

\footnotetext{
${ }^{7}$ Note that the transformation to probability space (from valuation space) is necessary for obtaining this linesegment interpretation.
} 


\subsection{The Ironed Algorithm}

We are now ready to define our ironed algorithm $\overline{\mathcal{A}}$. Given distribution $F$ and interval $I$, we will write $F[I]$ to mean $F$ restricted to $I$.

Definition 3.1 (Resample $(\mathcal{A}, \mathbb{I})$ ) Given algorithm $\mathcal{A}$ and a profile of disjoint interval sets, $\mathbb{I}=$ $\left\{\mathcal{I}_{1}, \ldots, \mathcal{I}_{n}\right\}$, the resampled algorithm for $\mathcal{A}$ with intervals $\mathbb{I}$ is algorithm $\operatorname{Resample}(\mathcal{A}, \mathbb{I})$ :

1. For each agent $i$, if $v_{i} \in I \in \mathcal{I}_{i}$, draw $\bar{v}_{i} \sim F_{i}[I]$; else, set $\bar{v}_{i}=v_{i}$.

2. Run $\mathcal{A}(\overline{\mathbf{v}})$.

Definition $3.2($ Monolnts $(\mathbf{x})$ ) The set of monotonizing intervals for $\mathbf{x}(\cdot)$ is MonoInts $(\mathbf{x})=$ $\left(\mathcal{I}_{1}, \ldots, \mathcal{I}_{n}\right)$ defined by:

1. Let $g_{i}(q)=x_{i}\left(F_{i}^{-1}(q)\right)$ be the allocation rule in probability space.

2. Let $G_{i}(q)=\int_{0}^{q} g_{i}(z) d z$ be the cumulative allocation rule.

3. Let $\bar{G}_{i}(\cdot)$ be the convex hull of $G_{i}(\cdot)$.

4. Let $\mathcal{I}_{i}$ be the set of intervals in valuation space on which $G_{i}\left(F_{i}(\cdot)\right)>\bar{G}_{i}\left(F_{i}(\cdot)\right)$.

Definition 3.3 $(\overline{\mathcal{A}})$ The ironed algorithm corresponding to algorithm $\mathcal{A}$ is the algorithm $\overline{\mathcal{A}}=$ $\operatorname{Resample}(\mathcal{A}, \operatorname{MonoInts}(\mathbf{x}))$.

Lemma $3.3 \overline{\mathcal{A}}$ is monotone.

Proof: We must show that each agent has a monotone allocation rule. The allocation rule for agent $i$ is precisely $\bar{x}_{i}\left(v_{i}\right)=\bar{g}\left(F_{i}\left(v_{i}\right)\right)$, which is the derivative of a convex function and therefore monotone.

Lemma 3.4 If $\mathcal{A}$ is a Bayesian $\beta$-approximation then $\overline{\mathcal{A}}$ is a Bayesian $\beta$-approximation.

Proof: First notice that the two allocation rules produce the same distribution over allocations and therefore expected costs are identical. We will show, for a single agent $i$, that $\mathbf{E}\left[v_{i} \bar{x}_{i}\left(v_{i}\right)\right] \geq$ $\mathbf{E}\left[v_{i} x_{i}\left(v_{i}\right)\right]$, from which linearity of expectation implies the result. We have

$$
\begin{aligned}
\mathbf{E}\left[v_{i} x_{i}\left(v_{i}\right)\right] & =\int_{0}^{1} v x_{i}(v) f_{i}(v) d v=\int_{0}^{1} F_{i}^{-1}(q) g_{i}(q) d q \\
& =\int_{0}^{1} \int_{0}^{F_{i}^{-1}(q)} g_{i}(q) d z d q=\int_{0}^{1} \int_{F_{i}(z)}^{1} g_{i}(q) d q d z \\
& =\int_{0}^{1}\left(G_{i}(1)-G_{i}\left(F_{i}(z)\right)\right) d z
\end{aligned}
$$

and similarly $\mathbf{E}\left[v_{i} \bar{x}_{i}\left(v_{i}\right)\right]=\int_{0}^{1}\left(\bar{G}_{i}(1)-\bar{G}_{i}\left(F_{i}(z)\right)\right) d z$. We conclude $\mathbf{E}\left[v_{i} \bar{x}_{i}\left(v_{i}\right)\right] \geq \mathbf{E}\left[v_{i} x_{i}\left(v_{i}\right)\right]$ since $\bar{G}_{i}(1)=G_{i}(1)$ and $\bar{G}_{i}\left(F_{i}(z)\right) \leq G_{i}\left(F_{i}(z)\right)$ for all $z \in[0,1]$.

Theorem 3.1 follows from Lemmas 3.3 and 3.4. 
Notes. We make the following notes about our main result. A more detailed discussion is given in our conclusions.

- The argument fails for non-linear objectives such as makespan. While D2 holds, it will not lead to an overall bound on the expected performance. See Appendix D for an example.

- Our ironing procedure is distinct from Myerson's, in the sense that Myerson's procedure yields a different mechanism. Myerson irons virtual valuations and allocates to maximize ironed virtual value. This is not the same as maximizing virtual value and then ironing the allocation rule where it is non-monotone. See Appendix A for an example.

- Even if $\mathcal{A}$ is a worst-case $c$-approximation, $\overline{\mathcal{A}}$ may fail to be a worst-case $c$-approximation. See Appendix $\mathrm{C}$ for an example.

\section{Reduction: Black-Box Model}

We now turn to a setting in which we do not have full functional access to allocation rules, but only black-box access to the given algorithm $\mathcal{A}$ and valuation distribution $\mathbf{F}$. We will use the ironing procedure from the previous section to monotonize an algorithm in this black-box model. Instead of using direct knowledge of the allocation rule, we must use sampling to estimate it. This sampling introduces errors in the selection of interval sets for resampling, which must then be dealt with. Our analysis will proceed in the following steps.

1. We describe a method for computing payments in the black-box model.

2. We describe a method for combining sampling with ironing to obtain a nearly monotone algorithm. In fact, this algorithm will be $\epsilon$-Bayesian incentive compatible.

3. We show that a convex combination of this nearly monotone algorithm with a blatantly monotone one will give a monotone algorithm, resulting in a BIC mechanism.

All of these steps approximately preserve social welfare. We obtain the following theorem.

Theorem 4.1 In the black-box model and general cost settings, for any $\epsilon>0$, a BIC algorithm $\mathcal{A}^{\prime}$ can be computed from any algorithm $\mathcal{A}$. Its expected social welfare satisfies $\mathcal{A}^{\prime} \geq \mathcal{A}-\epsilon$, and its runtime is polynomial in $n$ and $1 / \epsilon$.

The additive error in Theorem 4.1 can be converted into a multiplicative error whenever the expected welfare of $\mathcal{A}$ is not too small. We obtain the following corollary.

Corollary 4.1 In the black-box model and general cost settings, for any $\epsilon>0$, a BIC algorithm $\mathcal{A}^{\prime}$ can be computed from any algorithm $\mathcal{A}$. Its expected social welfare satisfies $\mathcal{A}^{\prime} \geq \mathcal{A} /(1+\epsilon)$, and its runtime is polynomial in $n, 1 / \epsilon$, and $1 / \mathcal{A}^{\prime}$.

Corollary 4.1 gives a construction with a multiplicative error in social welfare, but its runtime depends on the expected welfare of $\mathcal{A}$. In Appendix $\mathrm{F}$ we describe an improvement that removes this dependency, and implies a fully polynomial reduction for downward-closed settings.

In the remainder of this section we prove of Theorem 4.1. 


\subsection{Computing Payments}

Suppose that $\mathcal{A}$ has monotone allocation rules. The problem of designing a mechanism to implement $\mathcal{A}$ then reduces to calculating appropriate payments. These payments are completely determined by the allocation rule of $\mathcal{A}$, but in the black-box model we do not have direct access to the functional form of the allocation rule. Archer et al. [3] solve this problem by computing an unbiased estimator of the desired payment rule using only black-box calls to the algorithm. For completeness we now summarize their approach.

Definition 4.1 (black-box payments) If algorithm $\mathcal{A}$ does not allocate to agent $i$, then agent $i$ pays 0 . Otherwise, we compute the payment of agent $i$ as follows:

1. Choose $v_{i}{ }^{\prime}$ uniformly from $\left[0, v_{i}\right]$

2. Draw $\mathbf{v}_{-i}^{\prime} \sim \mathbf{F}_{-i}$ and $\operatorname{run} \mathcal{A}\left(v_{i}{ }^{\prime}, \mathbf{v}_{-i}^{\prime}\right)$

3. If $\mathcal{A}$ allocated to agent $i$ in the previous step set $X=v_{i}$, otherwise set $X=0$.

4. If $X \neq 0$, repeatedly draw values $\mathbf{v}_{-i}^{\prime} \sim \mathbf{F}_{-i}$ and run $\mathcal{A}\left(v_{i}, \mathbf{v}_{-i}^{\prime}\right)$ until the algorithm allocates to player $i$, and let $T$ be the number of iterations required.

5. Agent $i$ 's payment is $p_{i}=v_{i}-T X$.

As was shown by Archer et al., this computation attains the appropriate expected payment.

Claim 4.2 (Archer et al. [3]) In the black-box payment procedure, the expected payments are $p_{i}\left(v_{i}\right)=v_{i} x_{i}\left(v_{i}\right)-\int_{0}^{v_{i}} x_{i}(z) d z$.

We note that since we execute this procedure for agent $i$ only if he receives an allocation, which occurs with probability $x_{i}\left(v_{i}\right)$, the expected number of calls to $\mathcal{A}$ for each player is at most

$$
x_{i}\left(v_{i}\right)\left(1+\frac{1}{x_{i}\left(v_{i}\right)}\right) \leq 2 .
$$

Thus, in expectation, all payments can be computed with $2 n$ calls to $\mathcal{A}$.

Any mechanism paired with the above payment scheme will be individually rational (IR), meaning that a truthtelling agent will never obtain negative utility. This is true even if the allocation rule is not monotone. This follows immediately from the fact that the payment for an agent that declares value $v_{i}$ is never greater than $v_{i}$ (indeed, it is defined as $v_{i}$ minus a non-negative value).

\subsection{Sampling and $\epsilon$-Bayesian Incentive Compatibility}

We will be estimating the allocation rule of a non-monotone algorithm and attempting to iron it. This will fail to result in an absolutely monotone rule. In this section we show that a nearly monotone rule results in truthtelling as an $\epsilon$-Bayes-Nash equilibrium $(\epsilon$-BNE): the most an agent can gain from a non-truthtelling strategy is an additive $\epsilon$. We call such a mechanism $\epsilon$-Bayesian incentive compatible ( $\epsilon$-BIC).

Definition 4.2 ( $\epsilon$-BIC) A mechanism is $\epsilon$-Bayesian incentive compatible if truthtelling obtains at least as much utility as any other strategy, up to an additive $\epsilon$, assuming all other agents truthtell. That is, for all $i, v_{i}$, and $v^{\prime}, v_{i} x_{i}\left(v_{i}\right)-p_{i}\left(v_{i}\right) \geq v_{i} x\left(v^{\prime}\right)-p_{i}\left(v^{\prime}\right)-\epsilon$. 
The main theorem of this section is the following.

Theorem 4.2 In the black-box model and general cost settings, for any $\epsilon>0$, an $\epsilon$-BIC algorithm $\mathcal{A}^{\prime}$ can be computed from any algorithm $\mathcal{A}$. Its expected social welfare satisfies $\mathcal{A}^{\prime} \geq \mathcal{A}-\epsilon$, and its runtime is polynomial in $n$ and $1 / \epsilon$.

The resampling procedure from the previous section is the main workhorse for Theorem 4.2. The construction of algorithm $\mathcal{A}^{\prime}$ consists primarily of choosing interval sets on which to resample.

\subsection{1 $\epsilon$-closeness}

We now formalize a closeness property under which an allocation rule that is close to monotone is $\epsilon$-BIC (for some related $\epsilon$ ).

Definition 4.3 ( $\epsilon$-close) Given allocation rules $x(\cdot)$ and $x^{\prime}(\cdot)$ are $\epsilon$-close if $\left|x(v)-x^{\prime}(v)\right|<\epsilon$ for all $v$. Two algorithms or mechanisms are $\epsilon$-close if each agent's allocation rules are $\epsilon$-close.

Lemma 4.3 If non-monotone $\mathcal{A}^{\prime}$ is $\epsilon$-close to a monotone $\mathcal{A}$, then $\mathcal{A}^{\prime}$ is $(2 \epsilon)$-BIC.

Proof: Suppose agent $i$ is participating in $\mathcal{A}^{\prime}$ and has value $v_{i}$, but claims to have value $v_{i}{ }^{\prime}$. Assume $v_{i}>v_{i}{ }^{\prime}$; the opposite case is similar. Using the payment rule from Theorem 2.1, agent $i$ 's gain in utility from declaring $v_{i}{ }^{\prime}$ is:

$$
\left(v_{i} x_{i}{ }^{\prime}\left(v_{i}{ }^{\prime}\right)-p_{i}\left(v_{i}{ }^{\prime}\right)\right)-\left(v_{i} x_{i}{ }^{\prime}\left(v_{i}\right)-p_{i}\left(v_{i}\right)\right)=\left(v_{i}-v_{i}{ }^{\prime}\right) x_{i}{ }^{\prime}\left(v_{i}{ }^{\prime}\right)-\int_{v_{i}{ }^{\prime}}^{v_{i}} x_{i}{ }^{\prime}(z) d z .
$$

Since $x_{i}{ }^{\prime}(\cdot)$ is $\epsilon$-close to a monotone curve, it must be that $x_{i}{ }^{\prime}(z)+\epsilon \geq x_{i}{ }^{\prime}\left(v_{i}{ }^{\prime}\right)-\epsilon$ for all $z \in\left[v_{i}{ }^{\prime}, v_{i}\right]$. Thus $\int_{v_{i}{ }^{\prime}}^{v_{i}} x_{i}{ }^{\prime}(z) d z \geq\left(v_{i}-v_{i}{ }^{\prime}\right)\left(x_{i}{ }^{\prime}\left(v_{i}{ }^{\prime}\right)-2 \epsilon\right)$. This implies that the value in (1) is at most $2 \epsilon\left(v_{i}-v_{i}{ }^{\prime}\right)$, which is at most $2 \epsilon$.

Lemma 4.4 If $\mathcal{A}$ and $\mathcal{A}^{\prime}$ have the same expected costs ${ }^{8}$ and are $\epsilon$-close then $\mathcal{A}^{\prime} \geq \mathcal{A}-n \epsilon$.

Proof: For each agent $i, \mathbf{E}\left[v_{i} x_{i}{ }^{\prime}\left(v_{i}\right)\right] \geq \mathbf{E}\left[v_{i}\left(x_{i}\left(v_{i}\right)-\epsilon\right)\right] \geq \mathbf{E}\left[v_{i} x_{i}\left(v_{i}\right)\right]-\epsilon \mathbf{E}\left[v_{i}\right]$. The result then follows by linearity of expectation.

Lemma 4.5 If algorithms $\mathcal{A}$ and $\mathcal{A}^{\prime}$ are $\epsilon$-close, then for any collection $\mathbb{I}=\left\{\mathcal{I}_{1}, \ldots, \mathcal{I}_{n}\right\}$ of interval sets, resampled algorithms $\operatorname{Resample}(\mathcal{A}, \mathbb{I})$ and $\operatorname{Resample}\left(\mathcal{A}^{\prime}, \mathbb{I}\right)$ are $\epsilon$-close.

Proof: For any $i$, let $x_{i}$ and $x_{i}{ }^{\prime}$ be the allocation rules of $\mathcal{A}$ and $\mathcal{A}^{\prime}$, respectively. Let $\bar{x}_{i}$ and $\bar{x}_{i}{ }_{i}$ be the allocation rules of $\operatorname{Resample}(\mathcal{A}, \mathbb{I})$ and $\operatorname{Resample}\left(\mathcal{A}^{\prime}, \mathfrak{I}\right)$. Then for any $I \in \mathcal{I}_{i}$,

$$
\left|\bar{x}_{i}(I)-\bar{x}_{i}^{\prime}(I)\right|=\left|E_{v}[x(v) \mid v \in I]-E_{v}\left[x^{\prime}(v) \mid v \in I\right]\right|=\left|E_{v}\left[x(v)-x^{\prime}(v) \mid v \in I\right]\right|<\epsilon .
$$

Appropriate payments to turn an algorithm that is $\epsilon$-close to monotone into a mechanism that is $2 \epsilon$-BIC can be computed by the same process we would use for monotone algorithms.

\footnotetext{
${ }^{8}$ Recall that the expected cost of an algorithm $A$ with allocation rule $\mathbf{x}(\cdot)$ is $\mathbf{E}_{\mathbf{v} \sim \mathbf{F}}[c(\mathbf{x}(\mathbf{v}))]$.
} 


\subsubsection{Discretization}

A key step in our reduction will be in discretizing the allocation rules of the algorithm. This reduces the problem of estimating an allocation rule to estimating its value at a polynomial number of points. Moreover, our resulting allocation will not necessarily be monotone, but there will be only a polynomial number of points at which it can be non-monotone; we will use this to our advantage when fixing non-monotonicities in Section 4.3.

Definition 4.4 (Piecewise constant) An algorithm is $k$-piece piecewise constant if for each $i$ there is a partition of valuation space into at most $k$ intervals such that the allocation rule for agent $i$ is constant on each interval.

Definition $4.5\left(\operatorname{Disc}_{\epsilon}(\mathcal{A})\right)$ For a given $\epsilon>0$ and algorithm $\mathcal{A}$, the discretization of algorithm $\mathcal{A}, \operatorname{Disc}_{\epsilon}(\mathcal{A})$, is $\operatorname{Resample}(\mathcal{A}, \mathcal{I})$, where $\mathbb{I}=\left\{\mathcal{I}_{1}, \ldots, \mathcal{I}_{n}\right\}$ is the collection of intervals defined by

$$
\mathcal{I}_{i}=\{[0, \epsilon)\} \cup\left\{\left[\epsilon(1+\epsilon)^{t}, \epsilon(1+\epsilon)^{t+1}\right)\right\}_{0 \leq t \leq \log _{1+\epsilon}(1 / \epsilon)} .
$$

Lemma 4.6 $\operatorname{DiSc}_{\epsilon}(\mathcal{A})$ is $\log _{1+\epsilon}(1 / \epsilon)$-piece piecewise constant and $\operatorname{DiSC}_{\epsilon}(\mathcal{A}) \geq \mathcal{A}-2 n \epsilon$.

Proof: Let $\dot{\mathrm{x}}(\cdot)$ denote the allocation rules for $\operatorname{Disc}_{\epsilon}(\mathcal{A})$. The allocation curves for Disc $\epsilon(\mathcal{A})$ are constant on interval $[0, \epsilon)$ and all intervals of the form $\left[\epsilon(1+\epsilon)^{t}, \epsilon(1+\epsilon)^{t+1}\right)$, and there are at most $\log _{1+\epsilon}\left(\epsilon^{-1}\right)$ such intervals over the range $[\epsilon, 1]$. These intervals do, indeed, partition valuation space. Furthermore, $\mathbf{E}_{v_{i}}\left[v_{i} \dot{x}_{i}\left(v_{i}\right)\right] \geq(1-\epsilon) \mathbf{E}_{v_{i}}\left[v_{i} x_{i}\left(v_{i}\right)\right]-\epsilon \geq \mathbf{E}_{v_{i}}\left[v_{i} x_{i}\left(v_{i}\right)\right]-2 \epsilon$, as algorithm $\operatorname{DisC}_{\epsilon}(\mathcal{A})$ modifies any input value greater than $\epsilon$ by at most a factor of $(1-\epsilon)$. As the expected costs before and after discretization are the same, the result follows from linearity of expectation.

\subsubsection{Statistical Estimation}

We next describe a sampling procedure for estimating an allocation rule. This procedure will not form an algorithm, but rather generates an estimated allocation curve, which we will denote by $\mathbf{y}(\cdot)$. This estimate behaves like an allocation rule, but is not associated with an actual algorithm (and, in particular, need not be feasibly implementable).

Definition 4.6 (estimate allocation rule) Given algorithm $\mathcal{A}$ which is k-piece piecewise constant and $\epsilon>0$, an estimated allocation rule for $\mathcal{A}$ is a curve $\mathbf{y}(\cdot)$ found as follows:

1. for each agent $i$ and valuation-space piece $I_{j}$, draw $\frac{4}{\epsilon^{2}} \log (2 \mathrm{kn} / \epsilon)$ samples from $\mathbf{F}$ conditional on $v_{i} \in I_{j}$, and run $\mathcal{A}$ on each of these samples.

2. let $y_{i j}$ be the average allocation over the invocations to $\mathcal{A}$ above, for each $i$ and $j$.

3. Define $\mathbf{y}$ by $y_{i}(v)=y_{i j}$ for all $v \in I_{j}$

Lemma 4.7 If algorithm $\mathcal{A}$ is $k$-piece piecewise constant then, for any $\epsilon>0$, an estimated allocation rule $\mathbf{y}(\cdot)$ for $\mathcal{A}$ is $k$-piece piecewise constant, and is $\frac{\epsilon}{2}$-close to $\mathbf{x}(\cdot)$ with probability at least $1-\frac{\epsilon}{2}$. The number of black-box calls to $\mathcal{A}$ used in the construction of $\mathbf{y}(\cdot)$ is polynomial in $n, k$, and $1 / \epsilon$. 
Proof: The runtime bound and the fact that $\mathbf{y}(\cdot)$ is $k$-piece piecewise constant follow immediately from the definition. Choose some $i$ and let $I_{j}$ denote piece $j$ of the valuation space for agent $i$ in $\mathcal{A}$, and write $x_{i}\left(I_{j}\right)$ for the (constant) value of $x_{i}(v)$ for any $v \in I_{j}$. By the Hoeffding-Chernoff

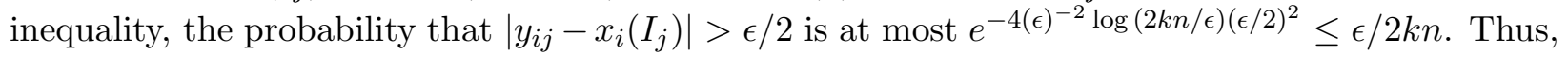
taking the union bound over all $i$ and $j$, we conclude that

$$
\left|y_{i j}-x_{i}\left(I_{j}\right)\right| \leq \frac{\epsilon}{2}
$$

for all $i$ and $j$ with probability at least $1-\frac{\epsilon}{2}$.

We now complete the proof of Theorem 4.2 by combining our sampling procedure with the ironing procedure from the ideal model.

Definition $4.7\left(\operatorname{IrON}_{\epsilon}(\mathcal{A})\right)$ Given piecewise constant algorithm $\mathcal{A}$, the statistically ironed algorithm for $\mathcal{A}$ with error $\epsilon>0$ is $\operatorname{IRON}_{\epsilon}(\mathcal{A})=\operatorname{Resample}(\mathcal{A}, \operatorname{MonoInts}(\mathbf{y}))$ where $\mathbf{y}(\cdot)$ is the estimated allocation rule for $\mathcal{A}$.

Note that $\operatorname{IrON}_{\epsilon}(\mathcal{A})$ is not simply a resampling of $\mathcal{A}$, but rather a convex combination of resamplings since the construction of interval set MonoInTs $(\mathbf{y})$ is randomized.

Lemma 4.8 $\operatorname{IrON}_{\epsilon}(\mathcal{A})$ is $2 \epsilon-B I C$ and $\operatorname{IrON}_{\epsilon}(\mathcal{A}) \geq \mathcal{A}-n \epsilon$.

Proof: By Lemma 4.7, $y_{i}(\cdot)$ is $k$-piece piecewise constant for each $i$. Let $\mathcal{A}_{\mathbf{y}}$ be the (fictional) algorithm with allocation rule $\mathbf{y}$. Since $\mathbb{I}$ is the monotonizing interval set for $\mathcal{A}_{\mathbf{y}}$, if $\mathcal{A}_{\mathbf{y}}$ were ironed according to $\mathbb{I}$, the result would be $\overline{\mathcal{A}}_{\mathbf{y}}$ which is monotone.

By Lemma 4.7, $\mathcal{A}_{\mathbf{y}}$ is $\frac{\epsilon}{2}$-close to $\mathcal{A}$ with probability $1-\frac{\epsilon}{2}$. In this case, Lemma 4.5 implies $\operatorname{Resample}(\mathcal{A}, \mathbb{I})$ is $\frac{\epsilon}{2}$-close to $\overline{\mathcal{A}}_{\mathbf{y}}$. For the remaining probability, $\frac{\epsilon}{2}$, we note that $\operatorname{Resample}(\mathcal{A}, \mathbb{I})$ is trivially 1-close to $\overline{\mathcal{A}}_{\mathbf{y}}$. Thus, taking expectation over all possible outcomes of the sampling, we conclude that $\operatorname{IRON}_{\epsilon}(\mathcal{A})$ is $\epsilon$-close to monotone, and is therefore $2 \epsilon$-BIC by Lemma 4.3.

Since, with probability $1-\frac{\epsilon}{2}, \operatorname{Resample}(\mathcal{A}, \mathbb{I})$ is $\frac{\epsilon}{2}$ close to $\overline{\mathcal{A}}_{\mathbf{y}}$ and $\mathcal{A}_{\mathbf{y}}$ is $\frac{\epsilon}{2}$ close to $\mathcal{A}$, Lemma 4.4 and Lemma 4.6 imply that, with probability $1-\frac{\epsilon}{2}$,

$$
\operatorname{Resample}(\mathcal{A}, \mathbb{I}) \geq \overline{\mathcal{A}}_{\mathbf{y}}-\frac{1}{2} n \epsilon \geq \mathcal{A}_{\mathbf{y}}-\frac{1}{2} n \epsilon \geq \mathcal{A}-n \epsilon
$$

For the remaining probability, $\frac{\epsilon}{2}$, we note that trivially $\operatorname{Resample}(\mathcal{A}, \mathbb{I}) \geq 0=\mathcal{A}-\mathcal{A} \geq \mathcal{A}-n$. Thus, taking expectation over all possible outcomes of sampling, we conclude $\operatorname{IRON}_{\epsilon}(\mathcal{A}) \geq \mathcal{A}-n \epsilon$.

Proof of Theorem 4.2: Define $\mathcal{A}^{\prime}$ to be the algorithm $\operatorname{IRON}_{\epsilon^{\prime}}\left(\operatorname{Disc}_{\epsilon^{\prime}}(\mathcal{A})\right)$, where $\epsilon^{\prime}=\epsilon / 3 n$. Then, by Lemmas 4.6 and $4.8, \mathcal{A}^{\prime}$ is $2 \epsilon^{\prime}$-BIC, and hence $\epsilon$-BIC, and $\mathcal{A}^{\prime} \geq \operatorname{Disc}_{\epsilon^{\prime}}(\mathcal{A})-n \epsilon^{\prime} \geq$ $\mathcal{A}-3 n \epsilon^{\prime}=\mathcal{A}-\epsilon$. The runtime of $\mathcal{A}^{\prime}$ (which is dominated by sampling in the construction of $\mathbf{y}$ ) is $\left.O\left(n k \epsilon^{\prime-2} \log \left(2 k n / \epsilon^{\prime}\right)\right)\right)=\tilde{O}\left(n^{3} \epsilon^{-3} \log \left(\epsilon^{-1}\right)\right)$, where recall $k=\frac{1}{\epsilon} \log (1 / \epsilon)$ is the number of discrete intervals in $\operatorname{DisC}_{\epsilon^{\prime}}(\mathcal{A})$.

\subsection{Bayesian Incentive Compatibility}

In the previous section we showed how to construct an $\epsilon$-BIC mechanism from any algorithm with almost no loss to the social welfare. Our goal now is to take such an $\epsilon$-BIC algorithm $\mathcal{A}$ and make it BIC. In other words, we would like to "fix" the (small) non-monotonicities in $\mathcal{A}$. Fortunately, since each allocation curve of $\mathcal{A}$ is discretized, any non-monotonicities must occur only at a small 
number of predetermined points. Our approach for removing these points of non-monotonicity is simple: we will construct an alternative algorithm $\mathcal{A}^{\prime}$ whose allocation curves are stair functions, with jumps in allocation probability occurring at each of those points. A convex combination of $\mathcal{A}$ and $\mathcal{A}^{\prime}$ will then be monotone. This convex combination will be our final BIC algorithm.

It is important that this convex combination process not reduce social welfare by too much. This requires two things. First, we need the convex combination to be mostly $\mathcal{A}$ as only it has provably good welfare. This is possible by taking $\epsilon$ so small that the explicit monotonicities in $\mathcal{A}^{\prime}$ heavily outweigh the non-monotonicities in $\mathcal{A}$ (which are at most $\epsilon$ ). Second, we need to ensure that the expected social welfare of $\mathcal{A}^{\prime}$ is not extremely negative.

How should we construct $\mathcal{A}^{\prime}$ ? Suppose first that we are in a downward-closed feasibility setting. In this case, the singleton allocation $\{i\}$ is feasible for each agent $i$. The construction of $\mathcal{A}^{\prime}$ with stair-function allocation curves is then straightforward: an agent $i$ is chosen uniformly at random and the algorithm then either allocates to agent $i$ or not, with the probability of allocation following a stair function. Since $\mathcal{A}^{\prime}$ only returns feasible outcomes, its expected social welfare must be nonnegative.

We would like to follow this same approach in general cost settings. However, it may be that, for some $i$, the particular allocation $\{i\}$ has an extremely high (or infinite) cost, in which case the above algorithm may have an extremely negative social welfare. Note, though, that in our construction we can replace $\{i\}$ with any allocation that includes agent $i$. It is therefore sufficient to find, for each $i$, some allocation that includes agent $i$ and whose cost is not too high. Once these allocations are found, we can use them to construct the stair algorithm $\mathcal{A}^{\prime}$.

In some cases finding low-cost allocations may be highly non-trivial. To get around this problem, we observe that as long as algorithm $\mathcal{A}$ has a reasonable probability of allocating to agent $i$, there must exist low-cost allocations that include $i$ that are returned by $\mathcal{A}$. We can therefore find such allocations by repeatedly sampling outcomes of $\mathcal{A}$. If, on the other hand, we were to take many samples and not find any allocations that include agent $i$, then we can safely assume that agent $i$ does not contribute much to the expected social welfare of $\mathcal{A}$. In this case, we can trivially monotonize agent $i$ 's allocation curve by ironing on interval $[0,1]$, removing the need to find allocations that include him.

\subsubsection{The Stair Algorithm}

We begin by demonstrating how to combine an $\epsilon$-BIC mechanism with an algorithm whose allocation rules are stair functions in order to obtain a BIC mechanism.

Definition $4.8(\operatorname{StaIR}(\mathcal{A}))$ Let $\mathcal{A}$ be a k-piece piecewise constant algorithm, and suppose $S_{1}, \ldots, S_{n}$ and $T_{1}, \ldots, T_{n}$ are allocations such that $i \in S_{i}$ and $i \notin T_{i}$ for all $i$. The stair algorithm for $\mathcal{A}$, $\operatorname{STAIR}(\mathcal{A})$, does the following:

1. Pick an agent $i$ uniformly from the $n$ agents.

2. If $v_{i}$ is in the $j$ th highest piece of $k$ pieces, allocate to $S_{i}$ with probability $(j-1) /(k-1)$ and $T_{i}$ otherwise.

Definition $4.9\left(\operatorname{ComB}_{\epsilon}(\mathcal{A})\right)$ Suppose algorithm $\mathcal{A}$ is k-piece piecewise constant. Then $\operatorname{ComB}_{\epsilon}(\mathcal{A})$ is the convex combination of $\mathcal{A}$ with probability $1-\delta$ and $\operatorname{STAIR}(\mathcal{A})$ with probability $\delta$, where $\delta=2(k-1) n \epsilon$. 
Lemma 4.9 If $\mathcal{A}$ is $\epsilon$-close to a monotone $\mathcal{A}^{\prime}$, then algorithm $\operatorname{Comb}_{\epsilon}(\mathcal{A})$ is $B I C$.

Proof: We will write $\hat{x}_{i}(\cdot)$ to denote an allocation rule of $\operatorname{ComB}_{\epsilon}(\mathcal{A})$. To show $\operatorname{ComB}_{\epsilon}(\mathcal{A})$ is BIC, choose any agent $i$ and any values $v_{i}<v_{i}{ }^{\prime}$; we will show $\hat{x}_{i}\left(v_{i}\right) \leq \hat{x}_{i}\left(v_{i}{ }^{\prime}\right)$. If $v_{i}, v_{i}{ }^{\prime}$ are in the same piece of the valuation space then $\hat{x}_{i}\left(v_{i}\right)=\hat{x}_{i}\left(v_{i}{ }^{\prime}\right)$. Otherwise, since $\mathcal{A}$ is $\epsilon$-close to monotone $\mathcal{A}^{\prime}$, it must be that $x_{i}\left(v_{i}\right) \leq x_{i}\left(v_{i}{ }^{\prime}\right)-2 \epsilon$. Furthermore, if $\mathbf{s}(\cdot)$ is the allocation rule for $\operatorname{STAIR}\left(\mathcal{A}^{\prime}\right)$, then $s_{i}\left(v_{i}\right) \leq s_{i}\left(v_{i}{ }^{\prime}\right)+1 /(k-1) n$. We conclude that

$$
\begin{aligned}
\hat{x}_{i}\left(v_{i}\right) & =(1-\delta) x_{i}{ }^{\prime}\left(v_{i}\right)+\delta s_{i}\left(v_{i}\right) \\
& \leq \hat{x}_{i}\left(v_{i}{ }^{\prime}\right)-2 \epsilon+\delta /(k-1) n \\
& =\hat{x}_{i}\left(v_{i}{ }^{\prime}\right)
\end{aligned}
$$

as required, since $\delta=2(k-1) n \epsilon$.

\subsubsection{Bounding Social Welfare: Finding Low-Cost Sets}

We now describe the choice of sets $S_{1}, \ldots, S_{n}$ and $T_{1}, \ldots, T_{n}$ for algorithm $\operatorname{StaIR}(\mathcal{A})$. What we require is that, for all $i, i \in S_{i}, i \notin T_{i}$, and $S_{i}, T_{i}$ are feasible (or have sufficiently low cost). In many settings finding such sets is trivial (e.g., for downward-closed feasibility problems we can take $S_{i}=\{i\}$ and $T_{i}=\emptyset$ ), but for some problems it might be difficult to find feasible (or low-cost) allocations. Our approach is as follows. Since $\mathcal{A}$ never makes an allocation that generates negative social welfare, we can bound the cost of any allocation made by $\mathcal{A}$. This motivates us to look for a set $S_{i} \ni i$ returned by $\mathcal{A}$ on some input, for each $i$. This can be accomplished by sampling. In the event that we do not find a set $S_{i}$, it is likely that the probability of allocating to agent $i$ is very low; we can therefore iron together all intervals for agent $i$, effectively removing the need for $S_{i}$, without causing much loss to the expected welfare. This operation can be viewed as trimming away agents that are very rarely allocated. The same holds for finding $T_{i}$.

Definition $4.10\left(\operatorname{TRIM}_{\epsilon}(\mathcal{A})\right)$ The trimmed algorithm for piece-wise constant $\mathcal{A}$ is $\operatorname{TRIM}_{\epsilon}(\mathcal{A})$ :

1. For each agent $i$ and valuation-space piece $I_{j} \in \mathcal{I}_{i}$, draw $\frac{4}{\epsilon^{2}} \log (2 n / \epsilon)$ samples from $\mathbf{F}$ conditional on $v_{i} \in I_{j}$, and run $\mathcal{A}$ on each of these samples.

2. If $\mathcal{A}$ is the same (always or never allocating) for $i$ on every sample, define $\mathcal{I}_{i}^{\prime}=\{[0,1]\}$; otherwise, $\mathcal{I}_{i}^{\prime}=\mathcal{I}_{i}$ and we define $S_{i}$ to be any observed allocation that includes agent $i$ and $T_{i}$ to be any observed allocation that does not include agent $i$.

3. Run $\operatorname{Resample}\left(\mathcal{A}, \mathbb{I}^{\prime}\right)$.

Note that, for each $i$, either sets $S_{i} \ni i$ and $T_{i} \not \supset i$ will be found during the execution of $\operatorname{TRIM}_{\epsilon}(\mathcal{A})$, or else the allocation rule of agent $i$ will be made constant.

Lemma 4.10 $\operatorname{TRIM}_{\epsilon}(\mathcal{A}) \geq \mathcal{A}-n \epsilon$.

Proof: We claim that, with probability at least $1-\frac{\epsilon}{2}$, for each agent $i$, the allocation rules for $\operatorname{TRIM}_{\epsilon}(\mathcal{A})$ and $\mathcal{A}$ will differ only on values $v_{i}$ for which $x_{i}\left(v_{i}\right) \leq \frac{\epsilon}{2}$. Before proving the claim, let us see how it implies the desired result. The claim implies that $\operatorname{TRIM}_{\epsilon}(\mathcal{A}) \geq \mathcal{A}-\left(\frac{\epsilon}{2}\right) n$ with probability 
$1-\frac{\epsilon}{2}$. For the remaining probability, we note that $\operatorname{TRIM}_{\epsilon}(\mathcal{A}) \geq 0=\mathcal{A}-\mathcal{A} \geq \mathcal{A}-n$ trivially. Thus, over all possible outcomes of sampling, we conclude that

$$
\operatorname{TRIM}_{\epsilon}(\mathcal{A}) \geq \mathcal{A}-\frac{\epsilon}{2} n-\frac{\epsilon}{2} n=\mathcal{A}-n \epsilon
$$

as required.

Let us now prove the claim. Choose some agent $i$ and $\operatorname{suppose}$ that $\operatorname{TRIM}_{\epsilon}(\mathcal{A})$ and $\mathcal{A}$ differ on some interval $I$ with $x_{i}(I) \geq \frac{\epsilon}{2}$. Then, by the definition of $\mathcal{I}_{i}^{\prime}$, it must be that no set $T \ni i$ was found during the sampling of interval $I$ for agent $i$. However, since $x_{i}(I) \geq \frac{\epsilon}{2}$, there is a probability of at least $\frac{\epsilon}{2}$ of finding such a set $T$ on each sample. By Chernoff-Hoeffding inequality, the probability that we do not find even one such set during $4 \epsilon^{-2} \log (2 n / \epsilon)$ samples is at most $\frac{\epsilon}{2 n}$. We conclude that the probability that no set $T \ni i$ was found during the sampling of interval $I$ is at most $\frac{\epsilon}{2 n}$. This is therefore a bound on the probability that $\operatorname{TRIM}_{\epsilon}(\mathcal{A})$ and $\mathcal{A}$ differ for agent $i$ on some interval $I$ with $x_{i}(I) \geq \frac{\epsilon}{2}$. By the union bound, the probability that this occurs for any agent is at most $\frac{\epsilon}{2}$, as required.

We are now ready to combine our tools into a BIC mechanism, proving Theorem 4.1.

Definition $4.11\left(\operatorname{MonO}_{\epsilon}(\mathcal{A})\right)$ Given an algorithm $\mathcal{A}$ and $\epsilon>0$, the monotonization of $\mathcal{A}$, denoted $\operatorname{MonO}_{\epsilon}(\mathcal{A})$, is the algorithm $\operatorname{ComB}_{\epsilon}\left(\operatorname{IrON}_{\epsilon}\left(\operatorname{TrIm}_{\epsilon}\left(\operatorname{Disc}_{\epsilon}(\mathcal{A})\right)\right)\right)$.

Lemma 4.11 $\operatorname{MonO}_{\epsilon}(\mathcal{A})$ is $B I C$, and $\operatorname{MonO}_{\epsilon}(\mathcal{A}) \geq \mathcal{A}-6 k n^{2} \epsilon$.

Proof: For notational convenience we define $\mathcal{A}^{\prime}=\operatorname{TRIM}_{\epsilon}\left(\operatorname{Disc}_{\epsilon}(\mathcal{A})\right)$. Recall that during the construction of $\mathcal{A}^{\prime}$ we find sets $S_{1}, \ldots, S_{n}$ with $S_{i} \ni i$. Also, Lemma 4.8 implies that $\operatorname{IrON}_{\epsilon}\left(\mathcal{A}^{\prime}\right)$ is $\epsilon$-close to a monotone algorithm. Thus $\operatorname{ComB}_{\epsilon}\left(\operatorname{IRON}_{\epsilon}\left(\mathcal{A}^{\prime}\right)\right)$ is well-defined, and is also BIC by Lemma 4.9.

Our ironing techniques do not affect the distribution of allocations generated by an algorithm, so the expected costs of $\operatorname{MonO}_{\epsilon}(\mathcal{A})$ and $\mathcal{A}$ are the same. Furthermore, by Lemmas 4.6, 4.8, and 4.10 ,

$$
\operatorname{IRON}_{\epsilon}\left(\mathcal{A}^{\prime}\right) \geq \mathcal{A}^{\prime}-n \epsilon=\operatorname{TRIM}_{\epsilon}\left(\operatorname{Disc}_{\epsilon}(\mathcal{A})\right)-n \epsilon \geq \operatorname{Disc}_{\epsilon}(\mathcal{A})-2 n \epsilon \geq \mathcal{A}-4 n \epsilon .
$$

We next claim that one can assume without loss of generality that $c\left(S_{i}\right) \leq n$ for all $i$. This is because $S_{i}$ is in the range of $\mathcal{A}$, and we can assume that $\mathcal{A}$ never returns an allocation that results in negative welfare (since otherwise a trivial improvement to $\mathcal{A}$ would return the empty allocation instead). Since valuations lie in $[0,1]$, non-negative welfare can be generated only by sets with cost at most $n$, and thus we can assume $c\left(S_{i}\right) \leq n$ for all $i$.

This implies that the expected social welfare obtained by $\operatorname{STAIR}\left(\operatorname{IRON}_{\epsilon}\left(\mathcal{A}^{\prime}\right)\right)$ is at least $(-n)$. We conclude

$$
\begin{aligned}
\operatorname{MonO}_{\epsilon}(\mathcal{A}) & =\operatorname{Comb}_{\epsilon}\left(\operatorname{Iron}_{\epsilon}\left(\mathcal{A}^{\prime}\right)\right) \\
& =(1-\delta) \operatorname{Iron}_{\epsilon}\left(\mathcal{A}^{\prime}\right)-\delta \operatorname{StaIR}\left(\operatorname{Iron}_{\epsilon}\left(\mathcal{A}^{\prime}\right)\right) \\
& \geq \mathcal{A}-4 n \epsilon-(2(k-1) n \epsilon) n \\
& \geq \mathcal{A}-6 k n^{2} \epsilon
\end{aligned}
$$

Proof of Theorem 4.1: Let $\mathcal{A}^{\prime}$ be the monotonized algorithm $\operatorname{MoNO}_{\epsilon^{\prime}}(\mathcal{A})$, where $\epsilon^{\prime}=\epsilon / 6 k n^{2}$. The result then follows immediately from Lemma 4.11. The runtime, which is dominated by sampling, is $O\left(k n\left(\epsilon^{\prime}\right)^{-2}\right)=\tilde{O}\left(\frac{n^{5}}{\epsilon^{5}} \log ^{3}(1 / \epsilon)\right)$. 


\section{Conclusions}

Our main result is for single-parameter agents and the objective of social welfare where we give a black-box reduction that converts any Bayesian approximation algorithm into a Bayesian incentive compatible mechanism. For these settings there is no gap separating the approximation complexity of algorithms and BIC mechanisms.

It is notable that our transformation from an approximation algorithm to a BIC mechanism cannot be duplicated by the agents acting on their own: there are non-monotone algorithms that, when coupled with any reasonable payment rule, do not have any BNE with near the expected welfare as the original algorithm on the true values. A concrete example is given in Appendix B.

While our main theorem is extremely general, the situations not covered by it are of notable interest.

1. Multi-parameter Bayesian mechanism design is not very well understood, but there is every reason to believe that approximation (which has not been pursued much by the economics literature) has a very interesting and relevant role to play in providing positive results. For the objetive of profit maximization the result of [10] reduces mechanism design to algorithm design in unit-demand settings with a natural "substitutability" property of the feasibility constraint, e.g., from matroid set systems. For social welfare maximization, the approach of this paper was recently generalized to convert any algorithm to a BIC mechanism in multidimensional discrete settings [16]. The same approach gives an $\epsilon$-BIC approximation in general multi-dimensional settings [7]. These reductions can be applied to the combinatorial public project problem for which Papadimitriou et al. [21] exhibit a gap separating the approximation complexity of algorithms and ex post IC mechanisms. These reductions show that the gap is in fact between BIC and IC mechanisms [7].

2. Our reduction applies to the objective of social welfare maximization. It would be nice to extend our result more generally to any monotone objective function, e.g., makespan. Unfortunately, our approach fails to preserve the approximation factor of the makespan objective. A concrete example that shows tha tour ideal reduction does not preserve expected makespan is given in Appendix D. Is there a polynomial-time reduction that turns any approximation algorithm for any monotone objective into a BIC mechanism with the same approximation factor?

3. In the special case where our reduction is applied to a worst-case $\beta$-approximation (recall: our reduction applies more generally to Bayesian $\beta$-approximations), the resulting BIC mechanism is still only a $\beta$-approximation in the weaker Bayesian sense. Is there a polynomial-time blackbox reduction that turns any worst-case $\beta$-approximation algorithm into a BIC mechanism that is also a worst-case $\beta$-approximation?

4. While Bayes-Nash equilibrium (i.e., BIC) is the standard equilibrium concept for implementation in economics, the stronger dominant strategy equilibrium (i.e., ex post IC) is the standard concept in computer science. The main challenge in obtaining a similar reduction for IC mechanisms is that the valuation space is exponentially big and monotonizing all points seems to require an exhaustive procedure. One potential approach would be to apply our ironing technique repeatedly, re-ironing an agent's curve whenever it is affected by an ironing of another agent's curve. Such a procedure will not generally give a monotone allocation 
rule; A concrete example is given in the full version of the paper. Is there a polynomialtime reduction for turning any $f(n)$-approximation (worst-case or Bayesian) algorithm for a single-parameter domain into an ex post IC mechanism that is a (worst-case or Bayesian) $\Theta(f(n))$-approximation?

The final question above is a refinement of what we consider to be the main open question of this work. Is there a gap separating the approximation complexity of implementation by Bayesian incentive compatible and ex post incentive compatible mechanisms for single-parameter social welfare maximization?

\section{References}

[1] G. Aggarwal and J. Hartline. Knapsack auctions. In Proceedings of the 17th Annual ACMSIAM Symposium on Discrete Algorithms, 2006.

[2] K. Akcoglu, J. Aspens, B. Dasgupta, and M. Kao. An opportunity-cost algorithm for combinatorial auctions. In Applied Optimization: Computational Methods in Decision-Making, Economics, and Finance, 2002.

[3] A. Archer, C. Papadimitriou, K. Talwar, and E. Tardos. An approximate truthful mechanism for combinatorial auctions with single parameter agents. In Proc. 14th ACM Symp. on Discrete Algorithms. ACM/SIAM, 2003.

[4] A. Archer and E. Tardos. Truthful mechanisms for one-parameter agents. In Proc. 42nd IEEE Symp. on Foundations of Computer Science, 2001.

[5] M. Babaioff and L. Blumrosen. Computationally-feasible truthful auctions for convex bundles. In Proc. 7th Intl. Workshop on Approximation Algorithms for Combinatorial Optimization Problems, 2004.

[6] M. Babaioff, R. Lavi, and E. Pavlov. Single-value combinatorial auctions and algorithmic implementation in undominated strategies. Journal of the ACM, 2009.

[7] X. Bei and Z. Huang. Bayesian incentive compatibility via fractional assignments. In Proc. 22st ACM Symp. on Discrete Algorithms, 2011.

[8] S. Bhattacharya, G. Goel, S. Gollapudi, and K. Munagala. Budget constrained auctions with heterogeneous items. In Proc. 41st ACM Symp. on Theory of Computing, 2010.

[9] A. Borodin and B. Lucier. Price of anarchy for greedy auctions. In Proc. 21st ACM Symp. on Discrete Algorithms, 2010.

[10] S. Chawla, J. Hartline, D. Malec, and B. Sivan. Sequential posted pricing and multi-parameter mechanism design. In Proc. 41st ACM Symp. on Theory of Computing, 2010.

[11] S. Chawla, J. Hartline, U. Rajan, and R. Ravi. Bayesian optimal no-deficit mechanism design. In Workshop on Internet and Network Economics (WINE), 2006.

[12] G. Christodoulou, A. Kovács, and Michael Schapira. Bayesian combinatorial auctions. In Proc. 35st Intl. Colloq. on Automata, Languages and Programming, pages 820-832, 2008. 
[13] P. Dhangwatnotai, S. Dobzinski, S. Dughmi, and T. Roughgarden. Truthful approximation schemes for single-parameter agents. In Proc. 49th IEEE Symp. on Foundations of Computer Science, 2008.

[14] J. Feigenbaum, C. Papadimitriou, and S. Shenker. Sharing the cost of multicast transmissions. Journal of Computer and System Sciences, 63(1):21-41, 2001.

[15] M. Gairing, B. Monien, and K. Tiemann. Selfish routing with incomplete information. In Proc. 17th ACM Symp. on Parallel Algorithms and Architectures, 2005.

[16] J. Hartline, R. Kleinberg, and A. Malekian. Bayesian incentive compatibility via matchings. In Proc. 22st ACM Symp. on Discrete Algorithms, 2011.

[17] R. Lavi. Computationally efficient approximation mechanisms. In N. Nisan, T. Roughgarden, É. Tardos, and V. Vazirani, editors, Algorithmic Game Theory, chapter 12, pages 301-329. Cambridge University Press, 2007.

[18] R. Lavi and C. Swamy. Truthful and near-optimal mechanism design via linear programming. In Proc. 46th IEEE Symp. on Foundations of Computer Science, 2005.

[19] D. Lehmann, L. I. O'Callaghan, and Y. Shoham. Truth revelation in approximately efficient combinatorial auctions. In Proc. 1st ACM Conf. on Electronic Commerce, pages 96-102. ACM Press, 1999.

[20] R. Myerson. Optimal auction design. Mathematics of Operations Research, 6:58-73, 1981.

[21] C. Papadimitriou, M. Schapira, and Y. Singer. On the hardness of being truthful. In Proc. 49th IEEE Symp. on Foundations of Computer Science, 2008.

\section{A Ironing Allocation Rules vs. Ironing Virtual Valuations}

At the heart of our mechanism construction is an ironing procedure that monotonizes allocation rules, outlined in Section 3. A similar process is used by Myerson as part of his construction of (revenue) optimal mechanisms for single-parameter settings [20]. In light of this similarity, we will now compare these two constructions and highlight their differences.

We first recall Myerson's optimal mechanism. For each agent $i$, the mechanism considers the virtual valuation function $\phi_{i}(\cdot)$ given by $\phi_{i}\left(v_{i}\right)=v_{i}-\frac{1-F_{i}\left(v_{i}\right)}{f_{i}\left(v_{i}\right)}$. This function is $\operatorname{monotonized}^{9}$ using the ironing method described in Section 3; the resulting monotone function is denoted $\overline{\phi_{i}}(\cdot)$. Given a valuation profile $\mathbf{v}$, the mechanism returns the allocation $\mathbf{x}$ that maximizes $\sum_{i} \overline{\phi_{i}}\left(v_{i}\right) \cdot x_{i}-$ $c(\mathbf{x})$. Myerson's celebrated result is that this allocation rule is revenue-optimal among the class of incentive compatible allocation rules.

Informally speaking, one can interpret Myerson's mechanism as first considering the allocation rule that maximizes social welfare with respect to the profile of virtual values $\phi_{i}\left(v_{i}\right)$. However, if the virtual valuation function is non-monotone, this allocation rule will also be non-monotone and hence not incentive compatible. The mechanism addresses this issue by ironing the virtual valuation function, which effectively monotonizes the allocation rule.

\footnotetext{
${ }^{9}$ Note that the virtual valuation function may be non-monotone if $F_{i}$ does not satisfy the monotone hazard rate assumption. For instance, bimodal distributions generally have non-monotone virtual valuation functions.
} 

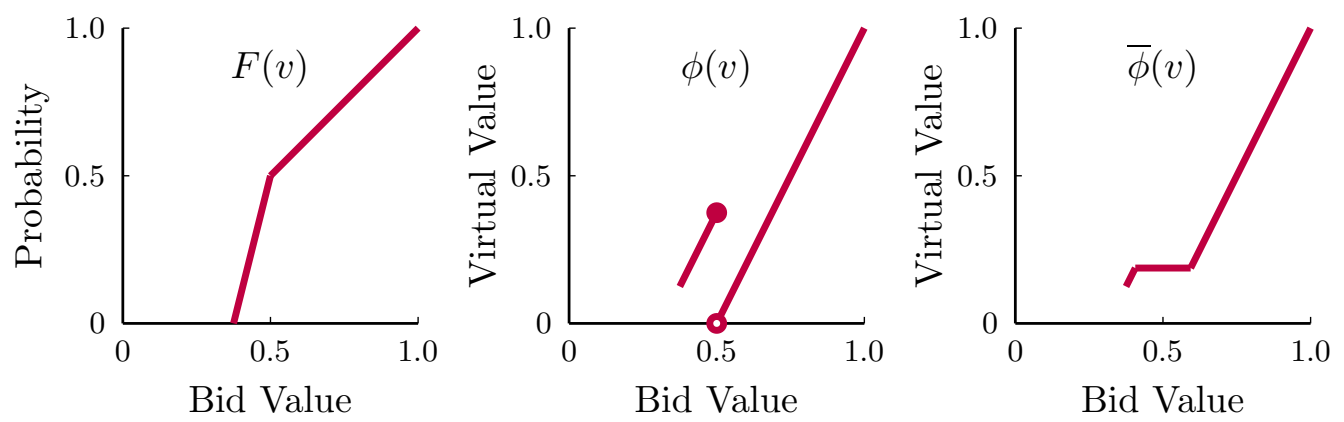

Figure 3: The distribution $F(v)$ used in Appendix A, with virtual valuation function $\phi(v)$ and ironed virtual valuation function $\bar{\phi}(v)$.

The motivation for ironing in our construction is quite similar, in that we are given a nonmonotone allocation rule that we wish to make incentive compatible. Furthermore, we address the issue in a similar way: by ironing the offending non-monotone curve. One might therefore suspect that these two monotonization procedures are, in fact, equivalent when restricted to the allocation rule that maximizes virtual welfare. However, as we will now show, this is not the case: the mechanisms that result from ironing the virtual valuation function and from ironing the allocation rule are distinct. Thus, our construction does differ, in an essential way, from that of Myerson.

Let us provide an example to illustrate this difference. Consider an auction of a single indivisible item to multiple bidders with values drawn i.i.d. from distribution $F$. Consider the following distribution $F$ : with probability $1 / 2$, the value is drawn uniformly from $\left[\frac{3}{8}, \frac{1}{2}\right]$; otherwise, it is drawn uniformly from $\left(\frac{1}{2}, 1\right]$ (see Figure 3 ). The virtual valuation function and ironed virtual valuation function corresponding to this distribution are

$$
\phi(v)=\left\{\begin{array}{ll}
2 v-\frac{5}{8} & v \in\left[\frac{3}{8}, \frac{1}{2}\right] \\
2 v-1 & v \in\left(\frac{1}{2}, 1\right]
\end{array} \quad \bar{\phi}(v)= \begin{cases}2 v-\frac{5}{8} & v \in\left[\frac{3}{8}, \frac{13}{32}\right] \\
\frac{3}{16} & v \in\left(\frac{13}{32}, \frac{19}{32}\right] \\
2 v-1 & v \in\left(\frac{19}{32}, 1\right] .\end{cases}\right.
$$

Suppose $\mathcal{A}$ is the allocation rule that assigns the item to the agent with highest virtual value. We now consider the two incentive compatible variants of $\mathcal{A}$ that we wish to compare. Namely, let $\mathcal{A}^{\prime}$ be Myerson's algorithm, which assigns the item to the agent with the highest ironed virtual value, and let $\overline{\mathcal{A}}$ be the ironed algorithm corresponding to $\mathcal{A}$ (as in Section 3 ). Let $x(\cdot), x^{\prime}(\cdot)$, and $\bar{x}(\cdot)$ denote the allocation curves corresponding to $\mathcal{A}, \mathcal{A}^{\prime}$, and $\overline{\mathcal{A}}$, respectively ${ }^{10}$. Our goal is to show that $\bar{x}(\cdot) \neq x^{\prime}(\cdot)$.

We observe that the function $\bar{\phi}(\cdot)$ achieves a strict minimum, over its effective range $\left[\frac{3}{8}, 1\right]$, at the point $v=\frac{3}{8}$. This implies that $x^{\prime}\left(\frac{3}{8}\right)=0$, since an agent that declares the minimal value can be awarded an allocation only in the 0-probability event that all other agents report this same value.

On the other hand, it must be that $\bar{x}\left(\frac{3}{8}\right)=\mathbf{E}_{v}[x(v) \mid v \leq z]$ for some $z \in\left[\frac{3}{8}, 1\right]$. We claim that this value is strictly positive. Indeed, $\phi(v)>\phi(w)$ for $v \in\left[\frac{3}{8}, \frac{1}{2}\right]$ and $w \in\left(\frac{1}{2}, \frac{9}{16}\right)$. This implies that $x(v)>0$ for all $v \in\left[\frac{3}{8}, \frac{1}{2}\right)$. We must therefore have $\bar{x}\left(\frac{3}{8}\right)=\mathbf{E}_{v}[x(v) \mid v \leq z]>0$.

We conclude $x^{\prime}\left(\frac{3}{8}\right) \neq \bar{x}\left(\frac{3}{8}\right)$, and thus the allocation rules $\mathcal{A}^{\prime}$ and $\overline{\mathcal{A}}$ are distinct.

\footnotetext{
${ }^{10}$ We drop the usual subscript of agent index since, by symmetry, the allocation curves are the same for each player.
} 


\section{B Equilibria of Non-Monotone Algorithms}

We have shown how to transform a non-monotone algorithm into a monotone one to obtain a mechanism that is BIC. It is notable that the agents could not do this on their own: there are nonmonotone algorithms that, when coupled with any individually-rational and no-positive-transfer ${ }^{11}$ payment rule, do not have any BNE with near the expected welfare as the original algorithm on the true values.

Choose parameter $X \gg n$. Consider an auction of a single indivisible item to $n$ bidders with values drawn i.i.d. from the following distribution: with probability $1 / n$ the value is $X$; with the remaining probability it is drawn uniformly from $[0,1]$. Let $\mathcal{A}$ allocate to the bidder with the largest value in $\left[0, \frac{1}{n^{2}}\right]$, if any and breaking ties randomly; otherwise it allocates to the bidder with the largest value.

Consider the expected welfare of $\mathcal{A}$. Since with high probability an agent has value $X$ and no agent has value $1 / n^{2}$ or below, $\mathcal{A}=\Omega(X)$.

Next we show that in any BNE most agents will bid $1 / n^{2}$ and the expected welfare will be the average value of the agents which is $O(X / n)$. Thus, the equiligrium is far from the algorithms Bayesian performance, i.e., the price of stability is linear.

Consider any mechanism that pairs $\mathcal{A}$ with an ex-post IR and no-positive-transfer payment scheme. We claim that in any BNE of such a mechanism, an agent with value greater than $\frac{1}{n^{2}}$ would instead report value $\frac{1}{n^{2}}$. To see this, consider a BNE and let $p$ denote the probability that some agent declares value $\frac{1}{n^{2}}$. Suppose that $p<1-\frac{1}{n}$. If agent $i$ has value $v_{i} \in\left[\frac{1}{8}, \frac{3}{8}\right]$ and he does not bid $\frac{1}{n^{2}}$, then his probability of allocation is at most $p(3 / 4+o(1))$ (since otherwise, with high probability, there will be an agent with value at least $2 v_{i}$ who could improve his utility by copying agent $i$ 's strategy). The expected utility of agent $i$ is therefore at most $p\left(3 v_{i} / 4+o(1)\right)$. On the other hand, agent $i$ could bid $\frac{1}{n^{2}}$ for an expected utility of at least $p\left(v_{i}-\frac{1}{n^{2}}\right)>p\left(v_{i} / 2+o(1)\right)$ (since $v_{i} \geq 1 / 8$ ). Thus any agent with a value in $\left[\frac{1}{8}, \frac{3}{8}\right]$ will bid $\frac{1}{n^{2}}$, so $p \geq 1-\frac{1}{n}$. We conclude by noting that if $p \geq 1-\frac{1}{n}$, every player with value above $\frac{1}{n^{2}}$ maximizes his utility by declaring $\frac{1}{n^{2}}$.

\section{Failure to Preserve Worst-Case Approximations}

We present an example to demonstrate that ideal ironing does not preserve worst-case approximation ratios. Consider an auction of 2 objects to 2 unit-demand bidders, with the goal of optimizing social welfare. The private value of agent 1 is drawn uniformly from $\{1,100\}$, and the private value of agent 2 is drawn uniformly from $\{10,1000,1001\}$. Let $\mathcal{A}$ be an approximation algorithm whose allocation rule is described in Figure 4.

We note that $\mathcal{A}$ is a worst-case 11/10 approximation algorithm (where the optimal solution is to always allocate to both players). Also, $\mathcal{A}$ is not BIC for agent $1: E\left[x_{1}(1)\right]=2 / 3$, whereas $E\left[x_{1}(100)\right]=1 / 3$. The ideal monotonization procedure will draw a new bid $v_{1}^{\prime}$ for agent 1 uniformly from $\{1,100\}$, and run $\mathcal{A}$ on $\left(v_{1}^{\prime}, v_{2}\right)$. Call this new algorithm $\overline{\mathcal{A}}$.

Note that if $v_{1}=100$ and $v_{2}=10$, then with probability $1 / 2 \overline{\mathcal{A}}$ will take $v_{1}^{\prime}=1$ and choose allocation $(0,1)$, and with the remaining probability it will take $v_{1}^{\prime}=100$ and choose allocation $(1,0)$. Hence, for this set of input values, the expected welfare obtained by $\overline{\mathcal{A}}$ is $\frac{100+10}{2}=55$. Since 110 is optimal, $\overline{\mathcal{A}}$ is at best a 2-approximation algorithm, whereas $\mathcal{A}$ is an $11 / 10$-approximation algorithm. We conclude that ideal ironing can cause a significant decrease in worst-case approximation ratios.

\footnotetext{
${ }^{11}$ No positive transfers implies that losers have zero payment.
} 


\begin{tabular}{c||c|c|c|} 
& 10 & 1000 & 1001 \\
\hline \hline 100 & 1,0 & 0,1 & 0,1 \\
\hline 1 & 0,1 & 1,1 & 1,1 \\
\hline
\end{tabular}

Figure 4: The allocation rule for algorithm $\mathcal{A}$. The vertical axis corresponds to $v_{1}$, the horizontal to $v_{2}$, and the table entries are of the form " $x_{1}, x_{2}$ ". For example, if $\left(v_{1}, v_{2}\right)=(100,10)$, then $\left(x_{1}, x_{2}\right)=(1,0)$.

\section{Beyond Social Welfare}

In the ideal model, our general reduction applies to any single-parameter optimization problem and converts an algorithm into a mechanism with at least the same expected social welfare. Unfortunately, this approach does not preserve other relevant objective values, even monotone ones such as the makespan. We illustrate this deficiency of the approach with an example.

Consider the problem of job scheduling on related machines where the objective is to minimize the makespan. Here the machines are agents and each has a (privately-known) speed. The time a job takes on a machine is the product of its length and the machine's speed. The goal of the mechanism is to assign a given set of jobs with varying lengths to the machines so as to minimize the time until all machines have finished processing their jobs, a.k.a., the makespan.

Consider an instance in which we have 10 unit-length jobs, and 5 machines. We assume a Bayesian setting, where the speeds of the machines are probabilistic. The first 4 machines are identical: they all have speed 2 with probability 1 . The last machine has either speed 1 or speed 2 , each with probability $1 / 2$.

Suppose $\mathcal{A}$ behaves in the following way. When $s_{5}=2$, it will choose $x_{i}=2$ for all $i$, resulting in a makespan of 1 . When $s_{5}=1, \mathcal{A}$ sets $\mathbf{x}=(6,1,0,0,3)$, resulting in a makespan of 3 (whereas the optimal is 1.5). Thus the average expected makespan achieved by $\mathcal{A}$ is 2 .

We note that, for this algorithm, the allocation curve for machine 5 is not monotone. Our monotonization procedure will therefore iron the valuation space of machine 5 . The optimal ironing of this curve will draw $s_{5}^{\prime}$ uniformly from $\{1,2\}$. This causes 4 equally likely possibilities, corresponding to $\left(s_{5}, s_{5}^{\prime}\right) \in\{1,2\}^{2}$.

If $s_{5}=s_{5}^{\prime}$ then $\mathcal{A}$ is proceeding as though no ironing occurred: if $s_{5}=s_{5}^{\prime}=1$ then the the makespan is 1 , and if $s_{5}=s_{5}^{\prime}=2$ the makespan is 3 . Suppose $s_{5}=2, s_{5}^{\prime}=1$. Then $\mathcal{A}$ forms allocation $x$ as though machine 5 has speed 1, though it actually has speed 2 . Hence we obtain $\mathbf{x}=(6,1,0,0,3)$, for a makespan of 3 . If, on the other hand, $s_{5}=1, s_{5}^{\prime}=2$ then we obtain $\mathbf{x}=(2,2,2,2,2)$, for a makespan of 2 .

We conclude that the expected makespan of the ironed procedure is $\frac{1+3+3+2}{4}=2.25$, which is strictly worse than the expected makespan obtained by the original algorithm.

\section{E Recursive Ironing does not Guarantee ex post IC}

In order for a mechanism to guarantee ex-post incentive compatibility, it must be that, for all $i \in[n]$, the allocation rule $x_{i}$ is monotone for any choice of $\mathbf{v}_{-i}$. Monotonizing each agent's allocation rule independently is insufficient to obtain this goal. Indeed, it is easy to construct examples where each each agent's allocation curve is monotone in expectation, but non-monotone for a particular 


\begin{tabular}{c||c|c|c|c|c|c} 
& 1 & 2 & 3 & 4 & 5 & 6 \\
\hline \hline 2 & 0.20 & 0.60 & 0.60 & 0.20 & 0.20 & 0.60 \\
\hline 1 & 0.80 & 0.20 & 0.82 & 0.22 & 0.84 & 0.24 \\
\hline
\end{tabular}

Figure 5: The allocation rule for algorithm $\mathcal{A}$. The vertical axis corresponds to possible values $v_{1}$, the horizontal axis corresponds to possible values $v_{2}$, and the table entries denote the probability of allocation to both agents. For example, if $\left(v_{1}, v_{2}\right)=(1,4)$, then $\left(x_{1}, x_{2}\right)=(0.22,0.22)$.

\begin{tabular}{c||c|c|c|c|c|c} 
& 1 & 2 & 3 & 4 & 5 & 6 \\
\hline \hline 2 & 0.40 & 0.40 & 0.40 & 0.40 & 0.40 & 0.40 \\
\hline 1 & 0.50 & 0.50 & 0.52 & 0.52 & 0.54 & 0.54 \\
\hline
\end{tabular}

(a)

\begin{tabular}{c||c|c|c|c|c|c|} 
& 1 & 2 & 3 & 4 & 5 & 6 \\
\hline \hline 2 & 0.45 & 0.45 & 0.46 & 0.46 & 0.47 & 0.47 \\
\hline 1 & 0.45 & 0.45 & 0.46 & 0.46 & 0.47 & 0.47 \\
\hline
\end{tabular}

Figure 6: The results of the recursive monotonization procedure for algorithm $\mathcal{A}$, on input $(1,5)$, (a) after 1 step, (b) after 2 steps.

choice of the other agents' bids.

One might imagine the following recursive approach for obtaining ex-post incentive compatibility. Begin by assuming that each agents' input is drawn from the singleton interval $I_{i}=\left[v_{i}, v_{i}\right]$, and let $\mathbf{I}$ denote the cube $I_{1} \times I_{2} \times \cdots \times I_{n}$. Choose some agent $i$ whose allocation curve is not monotone, under the assumption that each other agents' values are drawn from cube $\mathbf{I}$, and iron that agent's curve under this assumption. This ironing process may enlarge the interval from which agent $i$ 's value is drawn; update $I_{i}$ to be this new interval. Repeat this process, choosing a new agent on each iteration, until all agents' curves are monotone.

Unfortunately, as we now demonstrate, the above procedure fails to guarantee ex-post incentive compatibility. Consider an auction setting with 2 agents, such that either both agents receive an allocation or neither does. Suppose $\mathcal{A}$ is the algorithm with allocation rule described in Figure 5.

Consider the application of our recursive monotonization technique on this algorithm when $\left(v_{1}, v_{2}\right)=(1,5)$. Suppose we choose to monotonize the allocation curve for agent 2. Applying our monotonization procedure to the values $(0.8,0.2,0.82,0.22,0.84,0.24)$, we obtain ironed intervals $\{1,2\},\{3,4\}$, and $\{5,6\}$. The resulting allocation rule is shown in figure $6(\mathrm{a})$.

We next monotonize curve $x_{1}$ at the point $v_{2}=5$. This curve is non-monotone for agent 1 , and the resulting ironed interval is $\{1,2\}$. The resulting allocation rule is shown in figure 6(b). After this monotonization, the allocation curves for all agents are monotone. The final expected allocation probability for both players is 0.47 (the entry at $\left(v_{1}, v_{2}\right)=(1,5)$ ).

Next consider the application of this technique when $\left(v_{1}, v_{2}\right)=(2,5)$. Monotonizing agent 2 first, we apply our procedure to values $(0.2,0.6,0.6,0.2,0.2,0.6)$ and we obtained ironed interval $\{2,3,4,5\}$. The resulting allocation rule is shown in figure $7(\mathrm{a})$.

We next monotonize curve $x_{1}$ at the point $v_{2}=5$. This curve is non-monotone for agent 1 , and the resulting ironed interval is $\{1,2\}$. The resulting allocation rule is shown in figure $7(\mathrm{~b})$. After this monotonization, the allocation curve for agent 2 is no longer monotone, so we must iron again over the interval $\{1,2,3,4,5,6\}$. At this point all agents' allocation curves are monotone. The final expected allocation probability for both players is 0.46 .

What we have shown is that, given $v_{2}=5$, our recursive monotonization procedure generates 


\begin{tabular}{c||c|c|c|c|c|c} 
& 1 & 2 & 3 & 4 & 5 & 6 \\
\hline \hline 2 & 0.20 & 0.40 & 0.40 & 0.40 & 0.40 & 0.60 \\
\hline 1 & 0.80 & 0.52 & 0.52 & 0.52 & 0.52 & 0.24 \\
\hline
\end{tabular}

(a)

\begin{tabular}{c||c|c|c|c|c|c|} 
& 1 & 2 & 3 & 4 & 5 & 6 \\
\hline \hline 2 & 0.50 & 0.46 & 0.46 & 0.46 & 0.46 & 0.42 \\
\hline 1 & 0.50 & 0.46 & 0.46 & 0.46 & 0.46 & 0.42 \\
\hline
\end{tabular}

\begin{tabular}{c||c|c|c|c|c|c|} 
& 1 & 2 & 3 & 4 & 5 & 6 \\
\hline \hline 2 & 0.46 & 0.46 & 0.46 & 0.46 & 0.46 & 0.46 \\
\hline 1 & 0.46 & 0.46 & 0.46 & 0.46 & 0.46 & 0.46 \\
\hline \multicolumn{6}{c}{ (c) }
\end{tabular}

Figure 7: The results of the recursive monotonization procedure for algorithm $\mathcal{A}$, on input $(2,5)$, (a) after 1 step, (b) after 2 steps, (c) after 3 steps.

the allocation rule $x_{1}(1)=0.47>0.46=x_{1}(2)$ for agent 1 . This procedure therefore does not result in a monotone allocation rule, and hence does not obtain ex-post incentive compatibility.

\section{F Extension to General Valuations}

We now show how to modify our construction from Section 4 to obtain a multiplicative error for many problems of interest, such as downward-closed feasibility settings. To see how this contrasts with Theorem 4.1, consider a setting in which the expected valuation of each agent is exponentially small ${ }^{12}$. In this case, any additive error $\epsilon$ such that $\epsilon^{-1}$ is polynomial will dominate the expected welfare of algorithm $\mathcal{A}$. We therefore require a more general theorem in order to obtain meaningful results in this setting.

To this end, let $\mu_{\max }=\max _{i} \mathbf{E}\left[v_{i}\right]$ be the maximum expected valuation of any agent. The following is a tightened version of Theorem 4.1 in which the loss in social welfare is scaled by $\mu_{\max }$.

Theorem F.1 In the black-box model and general cost settings, for any $\epsilon>0$, a BIC algorithm $\mathcal{A}^{\prime}$ can be computed from any Bayesian algorithm $\mathcal{A}$. Its social welfare satisfies $\mathcal{A}^{\prime} \geq \mathcal{A}-\epsilon \mu_{\max }$, and its runtime is polynomial in $n, 1 / \epsilon$, and $\log \left(1 / \mu_{\max }\right)$.

For the special case of downward-closed set systems for feasibility problems, we can assume that $\mathcal{A} \geq \mu_{\max }$, since the trivial algorithm that simply allocates to the single player with the highest input value attains this value. This implies the following corollary.

Corollary F.1 In the black-box model and downward-closed feasibility settings, for any $\epsilon>0$, a BIC Bayesian $\beta(1+\epsilon)$-approximation algorithm $\mathcal{A}^{\prime}$ can be computed from any Bayesian $\beta$ approximation algorithm $\mathcal{A}$. Its runtime is polynomial in $n, 1 / \epsilon$, and $\log \left(1 / \mu_{\max }\right)$.

To prove Theorem F.1, we first consider the following variant of Theorem 4.2:

Theorem F.2 In the black-box model and general cost settings, for any $\epsilon>0$, an $\epsilon$-BIC algorithm $\mathcal{A}^{\prime}$ can be computed from any Bayesian algorithm $\mathcal{A}$. Its social welfare satisfies $\mathcal{A}^{\prime} \geq \mathcal{A}-\epsilon \mu_{\max }$, and its running time is polynomial in $n, 1 / \epsilon$, and $\log \left(1 / \mu_{\max }\right)$.

\footnotetext{
${ }^{12}$ Since values are scaled to lie in $[0,1]$, this situation can occur whenever the expected valuations of the agents are bounded, but agents can have exponentially larger values with positive probability.
} 
The proof of Theorem F.2 follows the proof of Theorem 4.2 from Section 4.2 almost exactly. Indeed, the only changes required are to replace instances of the inequality $\mathbf{E}\left[v_{i}\right] \leq 1$ with $\mathbf{E}\left[v_{i}\right] \leq$ $\mu_{\max }$ throughout, and to alter the definition of the discretization of an algorithm $\mathcal{A}$, Definition 4.5 , so that discretization occurs on the intervals

$$
\mathcal{I}_{i}=\left\{\left[0, \epsilon \mu_{\max }\right)\right\} \cup\left\{\left[\epsilon \mu_{\max }(1+\epsilon)^{t}, \epsilon \mu_{\max }(1+\epsilon)^{t+1}\right)\right\}_{0 \leq t \leq \log _{1+\epsilon}\left(1 / \epsilon \mu_{\max }\right)} .
$$

We omit further details of the proof of Theorem F.2.

We now turn to proving Theorem F.1 from Theorem F.2. Our approach will be the same as the proof of Theorem 4.1 from Theorem 4.2 in Section 4.3: we consider a convex combination of the almost-monotone algorithm from Theorem F.2 with the blatantly monotone stair algorithm. Recall that in Section 4.3 some care was necessary when finding sets $S_{1}, \ldots, S_{n}$. This task becomes much more difficult when we wish to keep our error bounded by $\epsilon \mu_{\max }$ (rather than $\epsilon$ ). We describe our approach in the next two subsections: we first give an algorithm for general cost settings, then present an optimization for the special case of feasibility settings.

\section{F.1 Implementing the Stair Algorithm in General Cost Settings}

In general cost settings, algorithm $\operatorname{STAIR}(\mathcal{A})$ may incur negative value if, for some $i$, the cost of set $S_{i}$ is large relative to $v_{i}$. To bound the expected welfare of $\operatorname{ComB}_{\epsilon}(\mathcal{A})$ we must therefore limit the costs of sets $S_{1}, \ldots, S_{n}$. Our upper bound on cost will depend on the following quantity, which relates to the structure of the piecewise constant intervals for algorithm $\mathcal{A}$.

Definition F.1 Suppose $\mathcal{A}$ has piece-wise constant allocation rules, where $\mathcal{I}_{i}=\left\{I_{1}, I_{2}, \ldots\right\}$ are the constant intervals for agent $i$. The stair threshold for agent $i, w_{i}^{\mathcal{A}}$, is defined as $w_{i}^{\mathcal{A}}:=\max I_{1}$ if $\left|\mathcal{I}_{i}\right|>1$; otherwise $w_{i}^{\mathcal{A}}:=\infty$. That is, $w_{i}^{\mathcal{A}}$ is the upper endpoint of the first valuation space interval for agent $i$, assuming the presence of multiple intervals.

We can now relate the value of $\operatorname{Comb}_{\epsilon}(\mathcal{A})$ to the cost of sets $S_{1}, \ldots, S_{n}$ and the stair thresholds of algorithm $\mathcal{A}$.

Lemma F.2 If there exists $X \geq 0$ such that $c\left(S_{i}\right) \leq w_{i}^{\mathcal{A}}+X$ for all $i$, then $\operatorname{ComB}_{\epsilon}(\mathcal{A}) \geq \mathcal{A}-$ $\delta\left(n \mu_{\max }+X\right)$.

Proof: $\quad$ By construction, $\operatorname{Comb}_{\epsilon}(\mathcal{A})=(1-\delta) \mathcal{A}+\delta \operatorname{STAIR}(\mathcal{A})$. Recall that $\operatorname{StaIR}(\mathcal{A})$ chooses some $i$ uniformly at random, and then either allocates $S_{i}$ or $\emptyset$. Moreover, $\operatorname{StaIR}(\mathcal{A})$ will always allocate $\emptyset$ if $v_{i}$ is in the first piece of the valuation space; that is, if $v_{i}<w_{i}^{\mathcal{A}}$. We therefore conclude that $\operatorname{StaIR}(\mathcal{A}) \geq \frac{1}{n} \sum_{i} \min \left\{w_{i}^{\mathcal{A}}-c\left(S_{i}\right), 0\right\} \geq-X$. Also, $\mathcal{A} \leq n \mu_{\text {max }}$ trivially. Thus $\left.\operatorname{Comb}_{\epsilon}(\mathcal{A}) \geq \mathcal{A}-\delta n \mu_{\max }+\delta(-X)\right)=\mathcal{A}-\delta\left(n \mu_{\max }+X\right)$.

Our goal will be to find sets $S_{i}$ with $c\left(S_{i}\right) \leq w_{i}^{\mathcal{A}}+n \mu_{\max } / \sqrt{\epsilon}$, then apply Lemma F.2 with $X=n \mu_{\max } / \sqrt{\epsilon}$. To find such sets, we will apply the same sampling techniques used in the construction of $\operatorname{IRON}_{\epsilon}(\mathcal{A})$. That is, for each $i$ and each piece of the valuation space, we will run $\mathcal{A}$ on many sample inputs. As long as $x_{i}(I)$ is not too small on a given interval $I$, we are very likely to find some valid allocation that includes agent $i$ during the sampling process! Moreover, we will show that not all sets discovered in this way can have high cost, so with high probability we will find a set $S_{i}$ with cost at most $n \mu_{\max } / \sqrt{\epsilon}$. To relate the cost of set $S_{i}$ with $w_{i}^{\mathcal{A}}$, we will also iron together all left-most intervals for which a low-cost set was not found. These ironed-together intervals will then act like a single piece of valuation space, which will allow us to relate the cost of any set $S_{i}$ we $d o$ find to the stair threshold for the (modified) algorithm. 
Definition F.2 $\left(\operatorname{TRIM}_{\epsilon}(\mathcal{A})\right)$ Given piece-wise constant algorithm $\mathcal{A}$, the stair-compatible algorithm for $\mathcal{A}, \operatorname{TRIM}_{\epsilon}(\mathcal{A})$, is as follows:

1. For each agent $i$ :

2. Let $\mathcal{I}_{i}=\left\{I_{1}, \ldots, I_{k}\right\}$ be the constant valuation space intervals for agent $i$.

3. For each $I_{j} \in \mathcal{I}_{i}$, draw $4 \epsilon^{-2} \log (2 n / \epsilon)$ samples from $\mathbf{F}$ conditional on $v_{i} \in I_{j}$, and run $\mathcal{A}$ on each of these samples.

4. Let $j_{i}$ be the minimal index such that, for some sample of interval $I_{j_{i}}$, $\mathcal{A}$ allocated a set $T_{i}$ with $T_{i} \ni i$ and $c\left(T_{i}\right) \leq \min I_{j_{i}}+n \mu_{\max } / \sqrt{\epsilon}$. Choose $S_{i}$ to be any such $T_{i}$.

5. If no such set $T_{i}$ was returned for any interval, take $j_{i}=k+1$ and $S_{i}=\{i\}$.

6. Define $\mathcal{I}_{i}^{\prime}=\left\{I_{1} \cup \ldots \cup I_{j_{i}-1}, I_{j_{i}}, \ldots, I_{k}\right\}$.

7. Run $\operatorname{Resample}\left(\mathcal{A}, \mathbb{I}^{\prime}\right)$.

Note that, as part of the execution of $\operatorname{TRIM}_{\epsilon}(\mathcal{A})$, a set $S_{i} \ni i$ will be found for each $i$, which is taken to be any set satisfying the conditions on line 4 for interval $I_{j_{i}}$ (or $\{i\}$ if no sets were found).

In summary, $\operatorname{TRIM}_{\epsilon}(\mathcal{A})$ samples each constant interval for agent $i$, searching for an appropriate set $S_{i}$. We take $I_{j_{i}}$ to be the leftmost interval for which such a set $S_{i}$ was found. All intervals to the left of $I_{j_{i}}$ are then ironed together. Thus, regardless of the sampling outcome, $I_{j_{i}}$ will be the second valuation space piece for agent $i$ in algorithm $\overline{\mathcal{A}}_{\mathbb{H}^{\prime}}$. Thus $c\left(S_{i}\right) \leq w_{i}^{\operatorname{TRIM}_{\epsilon}(\mathcal{A})}+n \mu_{\max } / \sqrt{\epsilon}$.

Lemma F.3 The stair compatible algorithm $\operatorname{TRIM}_{\epsilon}(\mathcal{A})$ for $\mathcal{A}$ (Definition F.2) and stair thresholds $\mathbf{w}^{\operatorname{TRIM}_{\epsilon}(\mathcal{A})}$ (Definition F.1) satisfy $c\left(S_{i}\right) \leq w_{i}^{\operatorname{TRIM}_{\epsilon}(\mathcal{A})}+n \mu_{\max } / \sqrt{\epsilon}$ for all $i$.

Proof: For each $i$, if no set satisfying the conditions on line 4 of $\operatorname{TRIM}_{\epsilon}(\mathcal{A})$ was found during the sampling of any interval, then $j_{i}=k+1$ and all intervals of $\mathcal{A}$ are ironed together in $\mathbb{I}^{\prime}$. In this case $w_{i}^{\operatorname{TRIM}_{\epsilon}(\mathcal{A})}=\infty$, so $c\left(S_{i}\right) \leq w_{i}^{\operatorname{TRIM}_{\epsilon}(\mathcal{A})}$ trivially. Otherwise, by line 4 of $\operatorname{TRIM}_{\epsilon}(\mathcal{A})$, $c\left(S_{i}\right) \leq \min I_{j_{i}}+n \mu_{\max } / \sqrt{\epsilon}$. However, since all intervals to the left of $I_{j_{i}}$ are ironed together in $\mathbb{I}^{\prime}, I_{j_{i}}$ will be the second piecewise constant interval of $\operatorname{TRIM}_{\epsilon}(\mathcal{A})$, and hence $\min I_{j_{i}}=w_{i}^{\operatorname{TRIM}_{\epsilon}(\mathcal{A})}$. Thus $c\left(S_{i}\right) \leq \min w_{i}^{\operatorname{TRIM}_{\epsilon}(\mathcal{A})}+n \mu_{\max } / \sqrt{\epsilon}$ as required.

Lemma F.4 $\operatorname{TRIM}_{\epsilon}(\mathcal{A}) \geq \mathcal{A}-2 n \mu_{\max } \sqrt{\epsilon}$.

Proof: We claim that, with probability at least $1-\frac{\epsilon}{2}$, for each agent $i$, the allocation rules for $\operatorname{TRIM}_{\epsilon}(\mathcal{A})$ and $\mathcal{A}$ will differ only on values $v_{i}$ for which $x_{i}\left(v_{i}\right) \leq \sqrt{\epsilon}+\frac{\epsilon}{2}$. Before proving the claim, let us see how it implies the desired result. The claim implies that $\operatorname{TRIM}_{\epsilon}(\mathcal{A}) \geq \mathcal{A}-\left(\sqrt{\epsilon}+\frac{\epsilon}{2}\right) n \mu_{\max }$ with probability $1-\frac{\epsilon}{2}$. For the remaining probability, we note that $\operatorname{TRIM}_{\epsilon}(\mathcal{A}) \geq \mathcal{A}-\mathcal{A} \geq \mathcal{A}-n \mu_{\max }$ trivially. Thus, over all possible outcomes of sampling, we conclude that

$$
\operatorname{TRIM}_{\epsilon}(\mathcal{A}) \geq \mathcal{A}-\left(\sqrt{\epsilon}+\frac{\epsilon}{2}\right) n \mu_{\max }-\frac{\epsilon}{2} n \mu_{\max } \geq \mathcal{A}-2 n \mu_{\max } \sqrt{\epsilon}
$$

as required. 
Let us now prove the claim. Choose some agent $i$ and suppose that $\operatorname{TRIM}_{\epsilon}(\mathcal{A})$ and $\mathcal{A}$ differ on some interval $I$ with $x_{i}(I) \geq \sqrt{\epsilon}+\frac{\epsilon}{2}$. Let $I$ be the leftmost such interval. For the remainder of the proof we will say that a set $T$ has low cost for $I$ if $c(T) \leq \min I+n \mu_{\max } / \sqrt{\epsilon}$. Then, by the definition of $\mathcal{I}_{i}^{\prime}$, it must be that no set $T \ni i$ with low cost was found during the sampling of interval $I$ for agent $i$. Let us bound the probability of this event. Given $\mathbf{v} \sim \mathbf{F}$, let $B(\mathbf{v})$ be the event $\left[x_{i}(\mathbf{v}) \wedge \sum_{i} v_{i} \leq \min I+n \mu_{\max } / \sqrt{\epsilon}\right]$. If event $B(\mathbf{v})$ occurs for some sample $\mathbf{v}$, this means that $\mathcal{A}$ returned some allocation $T \ni i$ and furthermore $\sum_{i} v_{i} \leq \min I+n \mu_{\max } / \sqrt{\epsilon}$. But note that this allocation must generate non-negative profit (otherwise it would never be allocated), and hence $T$ must have low cost for $I$. Thus $B(\mathbf{v})$ is precisely the event that $\mathcal{A}$ returns a set $T \ni i$ with low cost for $I$.

Consider the probability of $B(\mathbf{v})$. By Markov's inequality, $\operatorname{Pr}_{\mathbf{v}}\left[\sum_{j \neq i} v_{j}>n \mu_{\max } / \sqrt{\epsilon}\right]<\sqrt{\epsilon}$. Thus, since $v_{i} \geq \min I$ with probability 1 conditional on $v_{i} \in I, \operatorname{Pr}_{\mathbf{v}}\left[\sum_{i} v_{i}>\min I+n \mu_{\max } / \sqrt{\epsilon}\right]<$ $\sqrt{\epsilon}$. Also, $\operatorname{Pr}_{\mathbf{v}}\left[\neg x_{i}(\mathbf{v}) \mid v_{i} \in I\right]=1-x_{i}(I) \leq 1-\left(\sqrt{\epsilon}+\frac{\epsilon}{2}\right)$. The union bound then implies that $\operatorname{Pr}_{\mathbf{v}}[\neg B(\mathbf{v})] \leq 1-\left(\sqrt{\epsilon}+\frac{\epsilon}{2}\right)+\sqrt{\epsilon}=1-\frac{\epsilon}{2}$, so $\operatorname{Pr}_{\mathbf{v}}[B(\mathbf{v})] \geq \frac{\epsilon}{2}$.

By Chernoff-Hoeffding inequality, the probability that event $B$ does not occur even once during $\epsilon^{-2} \log (2 n / \epsilon)$ samples is at most $\frac{\epsilon}{2 n}$. We conclude that the probability that no set $T \ni i$ with low cost was found during the sampling of interval $I$ is at most $\frac{\epsilon}{2 n}$. This is therefore a bound on the probability that $\operatorname{TRIM}_{\epsilon}(\mathcal{A})$ and $\mathcal{A}$ differ for agent $i$ on some interval $I$ with $x_{i}(I) \geq \sqrt{\epsilon}+\frac{\epsilon}{2}$. By the union bound, the probability that this occurs for any agent is at most $\frac{\epsilon}{2}$, as required.

We are now ready to describe the algorithm used to prove Theorem F.1.

Definition F.3 $\left(\operatorname{MonO}_{\epsilon}(\mathcal{A})\right)$ Given an algorithm $\mathcal{A}$ and any $\epsilon>0$, the monotonization of $\mathcal{A}$, $\operatorname{Mono}_{\epsilon}(\mathcal{A})$, is $\operatorname{Comb}_{\epsilon}\left(\operatorname{IrON}_{\epsilon}\left(\operatorname{TRIM}_{\epsilon}\left(\operatorname{Disc}_{\epsilon}(\mathcal{A})\right)\right)\right)$.

Lemma F.5 $\operatorname{Mono}_{\epsilon}(\mathcal{A})$ is $B I C$, and $\operatorname{MonO}_{\epsilon}(\mathcal{A}) \geq \mathcal{A}-9 k n^{2} \sqrt{\epsilon} \mu_{\max }$.

Proof: For notational convenience define $\mathcal{A}^{\prime}=\operatorname{TRIM}_{\epsilon}\left(\operatorname{Disc}_{\epsilon}(\mathcal{A})\right)$. Lemma 4.8 implies that $\operatorname{IRON}_{\epsilon}\left(\mathcal{A}^{\prime}\right)$ is $\epsilon$-close to a monotone algorithm, and during the construction of $\mathcal{A}^{\prime}$ we find sets $S_{1}, \ldots, S_{n}$ with $S_{i} \ni i$. Thus $\operatorname{ComB}_{\epsilon}\left(\operatorname{IRON}_{\epsilon}\left(\mathcal{A}^{\prime}\right)\right)$ is well-defined and, by Lemma 4.9, is BIC.

We note that costs are not affected by our ironing techniques, and, by Lemma F.4,

$$
\begin{aligned}
\operatorname{IRON}_{\epsilon}\left(\mathcal{A}^{\prime}\right) & \geq \mathcal{A}^{\prime}-n \epsilon \mu_{\max }=\operatorname{TRIM}_{\epsilon}\left(\operatorname{Disc}_{\epsilon}(\mathcal{A})\right)-n \epsilon \mu_{\max } \\
& \geq \operatorname{Disc}_{\epsilon}(\mathcal{A})-n \epsilon \mu_{\max }-2 n \mu_{\max } \sqrt{\epsilon} \geq \mathcal{A}-5 \sqrt{\epsilon} n \mu_{\text {max }}
\end{aligned}
$$

Also, $c\left(S_{i}\right) \leq w_{i}^{\mathcal{A}^{\prime}}+n \mu_{\max } / \sqrt{\epsilon} \leq w_{i}^{\mathrm{IRON}_{\epsilon}\left(\mathcal{A}^{\prime}\right)}+n \mu_{\max } / \sqrt{\epsilon}$ for all $i$ by Lemma F.3. Thus, by Lemma F.2,

$$
\begin{aligned}
\operatorname{MONO}_{\epsilon}(\mathcal{A}) & =\operatorname{Comb}_{\epsilon}\left(\operatorname{IRON}_{\epsilon}\left(\mathcal{A}^{\prime}\right)\right) \\
& \geq \operatorname{IRON}_{\epsilon}\left(\mathcal{A}^{\prime}\right)-\delta\left(n \mu_{\max }+n \mu_{\max } / \sqrt{\epsilon}\right) \\
& \geq \mathcal{A}-5 n \sqrt{\epsilon} \mu_{\max }-(2(k-1) n \epsilon) 2 n \mu_{\max } / \sqrt{\epsilon} \\
& \geq \mathcal{A}-9 k n^{2} \sqrt{\epsilon} \mu_{\max } .
\end{aligned}
$$

Theorem F.1 now follows immediately from Lemma F.5 by considering algorithm $\operatorname{MoNO}_{\epsilon^{\prime}}(\mathcal{A})$, where $\epsilon^{\prime}=\left(\epsilon / 9 k n^{2}\right)^{2}=\epsilon^{2} / 81 k^{2} n^{4}$. The runtime, which is dominated by sampling, is $O\left(k n\left(\epsilon^{\prime}\right)^{-2}\right)=$ $\tilde{O}\left(n^{9} \epsilon^{-9} \log ^{5}\left(v_{\max } / \epsilon \mu_{\max }\right)\right)$. 


\section{F.2 Feasibility Settings}

In general feasibility settings, where costs are either 0 or infinite, the performance of algorithm $\operatorname{MonO}_{\epsilon}(\mathcal{A})$ improves significantly. Specifically, we can improve Lemma F.4 as follows:

Lemma F.6 In feasibility settings, $\operatorname{TRIM}_{\epsilon}(\mathcal{A}) \geq \mathcal{A}-\epsilon n \mu_{\max }$.

Proof: Consider some agent $i$ and interval $I \in \mathcal{I}$, and suppose $x_{i}(I) \geq \frac{\epsilon}{2}$. Consider the probability of finding an allocation with cost at most $\min I+n \mu_{\max } / \sqrt{\epsilon}$ when sampling for this interval. Since costs are either 0 or $\infty$, this is precisely the probability of finding an allocation that includes agent $i$, which is $x_{i}(I) \geq \frac{\epsilon}{2}$. By Chernoff-Hoeffding inequality, the probability that this event does not occur even once in $\epsilon^{-2} \log (n / 2 \epsilon)$ samples is at most $\epsilon / 2 n$. We will therefore successfully find a set $S_{i} \ni i$ with probability at least $1-\epsilon / 2 n$.

For each $i$, let $I_{j_{i}}$ denote the leftmost interval on which $x_{i}(I) \geq \epsilon$. By the union bound, with probability $1-\epsilon / 2$ we will find a set $S_{i} \ni i$ when sampling interval $I_{j_{i}}$, for all $i$. In this case, the behavior of algorithms $\operatorname{TRIM}_{\epsilon}(\mathcal{A})$ and $\mathcal{A}$ differ only on intervals $I$ to the left of $I_{j_{i}}$, all of which satisfy $x_{i}(I)<\frac{\epsilon}{2}$. Thus, conditioning on an event of probability $1-\frac{\epsilon}{2}, \operatorname{TRIM}_{\epsilon}(\mathcal{A}) \geq \mathcal{A}\left(1-\frac{\epsilon}{2}\right) \geq$ $\mathcal{A}-n \mu_{\max } \epsilon$. For the remaining probability, $\frac{\epsilon}{2}$, we note that $\mathcal{A} \leq n \mu_{\max }$ trivially. We conclude that $\mathcal{A}_{\epsilon} \geq \mathcal{A}-\epsilon n \mu_{\max }$ unconditionally.

Using Lemma F.6 instead of Lemma F.4 in the analysis of $\operatorname{MoNO}_{\epsilon}(\mathcal{A})$, we find that the statement of Lemma F.5 improves to show that $\operatorname{MoNO}_{\epsilon}(\mathcal{A})>\mathcal{A}-8 k n^{2} \epsilon \mu_{\max }$ in feasibility settings. Thus, in feasibility settings, we can improve the runtime of the algorithm in Theorem F.1 by taking $\mathcal{A}^{\prime}$ to be $\operatorname{MonO}_{\epsilon^{\prime}}(\mathcal{A})$ with $\epsilon^{\prime}=8 k n^{2} \epsilon$, which has a runtime of $O\left(k n\left(\epsilon^{\prime}\right)^{-2}\right)=\tilde{O}\left(n^{5} \epsilon^{-5} \log ^{3}\left(v_{\max } / \epsilon \mu_{\max }\right)\right)$. 